\title{
FLORA Y VEGETACIÓN DE LAS ZONAS NÚCLEO DE LA RESERVA DE LA Biosfera Mariposa Monarca, México
}

\author{
Guadalupe Cornejo-Tenorio ${ }^{1}$, Alejandro Casas ${ }^{1}$, Berenice Farfán ${ }^{1}$, José Luis \\ VILLASEÑOR ${ }^{2}$ Y GUILlermo IBARRA-MANRÍQUeZ ${ }^{1,3}$
}

${ }^{1}$ Centro de Investigaciones en Ecosistemas, Universidad Nacional Autónoma de México, Antigua carretera a Pátzcuaro No. 8701, Col. San José de la Huerta, C.P. 58190, Morelia, Michoacán, México.

2 Departamento de Botánica, Instituto de Biología, Universidad Nacional Autónoma de México, Ap. Postal 70-367, Ciudad Universitaria, México 04510, D.F.

${ }_{3}^{3}$ Autor para la correspondencia. Correo-e: gibarra@oikos.unam.mx

\begin{abstract}
Resumen: Se realizó un estudio florístico y fisonómico de la vegetación en las zonas núcleo de la Reserva de la Biosfera Mariposa Monarca en los estados de México y Michoacán. Se efectuaron colectas botánicas durante un ciclo anual y se realizó una revisión de material previamente recolectado en la zona, depositado en los herbarios mexicanos IEB, EBUM y MEXU. El listado florístico incluye 423 especies que incluyen 32 taxa infraespecíficos de plantas vasculares, agrupadas en 87 familias y 244 géneros. Las familias con mayor riqueza de especies fueron Asteraceae (103), Lamiaceae (21), Fabaceae (17) y Scrophulariaceae (17), en tanto que a nivel de género sobresalen Salvia (13), Stevia (8) y Ageratina (7). Un total de 135 especies (31.9\%), que incluyen 13 taxa infraespecíficos, son endémicas de México; 40 de estas especies pertenecen a Asteraceae. Las hierbas fueron la forma de crecimiento más frecuente (326 especies), seguidas por los arbustos (51) y los árboles (33). Se reconocen principalmente los siguientes tipos de vegetación: bosque de coníferas, bosque de Quercus, bosque mesófilo de montaña y pastizal antropogénico.
\end{abstract}

Palabras clave: florística, México, Reserva de la Biosfera Mariposa Monarca, vegetación.

\begin{abstract}
A study of the flora and vegetation physiognomy was conducted at the core zones of the Monarch Butterfly Biosphere Reserve, in the states of México and Michoacán. Collection of plant specimens was carried out during one year of field work and this activity was supplemented by a review of voucher specimens previously collected in the area and deposited at the Mexican herbaria IEB, EBUM, and MEXU. A total of 423 vascular plant species including 32 infraespecific taxa and grouped in 86 families and 244 genera were identified. Families with the largest species richness were Asteraceae (103), Lamiaceae (21), Fabaceae (17), and Scrophulariaceae (17). The most speciose genera were Salvia (13), Stevia (8), and Ageratina (7). Mexican endemic species comprised 135 (31.9\%) species, which included 13 infraespecific taxa; 40 of these species belong to Asteraceae. Herbs were represented by 326 species, followed by shrubs (51) and trees (33). The main vegetation types recognized were coniferous forest (including combinations of fir, pine, and oak forests), cloud forest, anthropogenic grassland, and Quercus forest.
\end{abstract}

Key words: floristic, Mexico, Monarch Butterfly Biosphere Reserve, vegetation.

$\mathbf{L}$ a Reserva de la Biosfera Mariposa Monarca (RBMM), ubicada en los estados de México y Michoacán, forma parte del Eje Volcánico Transversal (el cual es denominado Faja Volcánica Transmexicana por Ferrusquía-Villafranca 1993), una provincia fisiográfica que resalta por su importancia biológica en cuanto a riqueza de especies y endemismo de anfibios, aves, briofitas, fanerógamas, mamíferos y reptiles (Rzedowski, 1991a; Delgadillo, 1993; Escalante-Pliego et al., 1993; Fa y Morales, 1993; FloresVillela, 1993; Nixon, 1993; Styles, 1993; Delgadillo et al., 2003). Esta provincia es muy interesante desde una perspectiva fitogeográfica, ya que en ella confluyen, sin límites muy precisos, elementos pertenecientes a los reinos biogeográficos Holártico y Neotropical o Sudamericano (Rzedowski, 1978; Cox, 2001). En el área predominan tipos de vegetación templada, especialmente bosques de coníferas y de encinos (Rzedowski, 1978), para los que se ha sugerido que albergan aproximadamente 7,000 especies a lo largo de su distribución en México, 70\% de las cuales son endémicas del país (Rzedowski, 1991a).

La RBMM es mundialmente famosa por ser el sitio donde ocurre la hibernación invernal de la mariposa monarca 
Danaus plexippus L. (Broker, 1999), cuyas poblaciones viajan desde el sur de Canadá, constituyendo uno de los fenómenos biológicos de migración contemporáneos más sorprendentes. Sin embargo, la reserva también se caracteriza por ser el escenario de complejos problemas económicos, sociales y políticos que inciden en un manejo inadecuado de los recursos naturales que contiene (Melo-Gallegos y López-García, 1989; Alonso y Alonso, 1999; Bernal-Hernández y Camacho-Vera, 1999; Merino, 1999), lo que pone en peligro su viabilidad como área prioritaria de conservación.

Para el establecimiento de esta área como Reserva de la Biosfera se elaboró un programa de manejo (SEMARNAT, 2001) que contempla como uno de sus puntos medulares el inventario de los recursos naturales que existen en la zona, incluyendo listas preliminares de flora y fauna. Para documentar de manera más precisa la riqueza florística de la RBMM, los estudios florísticos y de vegetación son una contribución fundamental. Un trabajo pionero al respecto fue el de Ibarra (1983), en el que se elaboró un listado florístico de 300 especies del Cerro Cacique. Posteriormente se han realizado estudios donde se describe la estructura y se mencionan las especies dominantes de los tipos de vegetación, principalmente para la Sierra de Chincua, los cuales incluyen listas florísticas (Espejo-Serna et al., 1992; Soto-Núñez y Vázquez-García, 1993; Mejía-Mendoza, 1996; Pinto-León, 2000; Genchi, 2002).

La información generada en estos trabajos refleja un esfuerzo notable por determinar la riqueza florística de la RBMM, la cual podría ser de al menos 800 especies. Sin embargo, varios de los listados mencionados carecen de ejemplares de referencia en herbarios nacionales, lo que impide cuantificar con mayor certeza las especies que existen en esta área protegida. Uno de los problemas más relevantes es que la determinación de las especies no puede ser verificada, un asunto que no es trivial si se considera la complejidad de muchos de los taxa vegetales presentes en el área (p.ej. Asteraceae, Poaceae, Salvia, Senecio, o Quercus). Otro inconveniente no menos importante es la imposibilidad de realizar actualizaciones nomenclaturales de los nombres de las especies, un proceso continuo cuya realización adecuada requiere por lo general de material de herbario. Tomando en cuenta lo anterior, el presente trabajo tiene por objetivo contribuir al conocimiento de la flora de las zonas núcleo de la RBMM, por medio de un listado actualizado de las plantas vasculares localizadas dentro de su territorio, así como describir sus principales tipos de vegetación con base en su fisonomía y composición florística.

\section{Área de estudio}

La RBMM se localiza en los municipios de Angangueo, Aporo, Contepec, Ocampo, Senguio y Zitácuaro en el estado de Michoacán, y en los municipios de Donato Guerra, San Felipe del Progreso, Temascalcingo y Villa de Allende en el

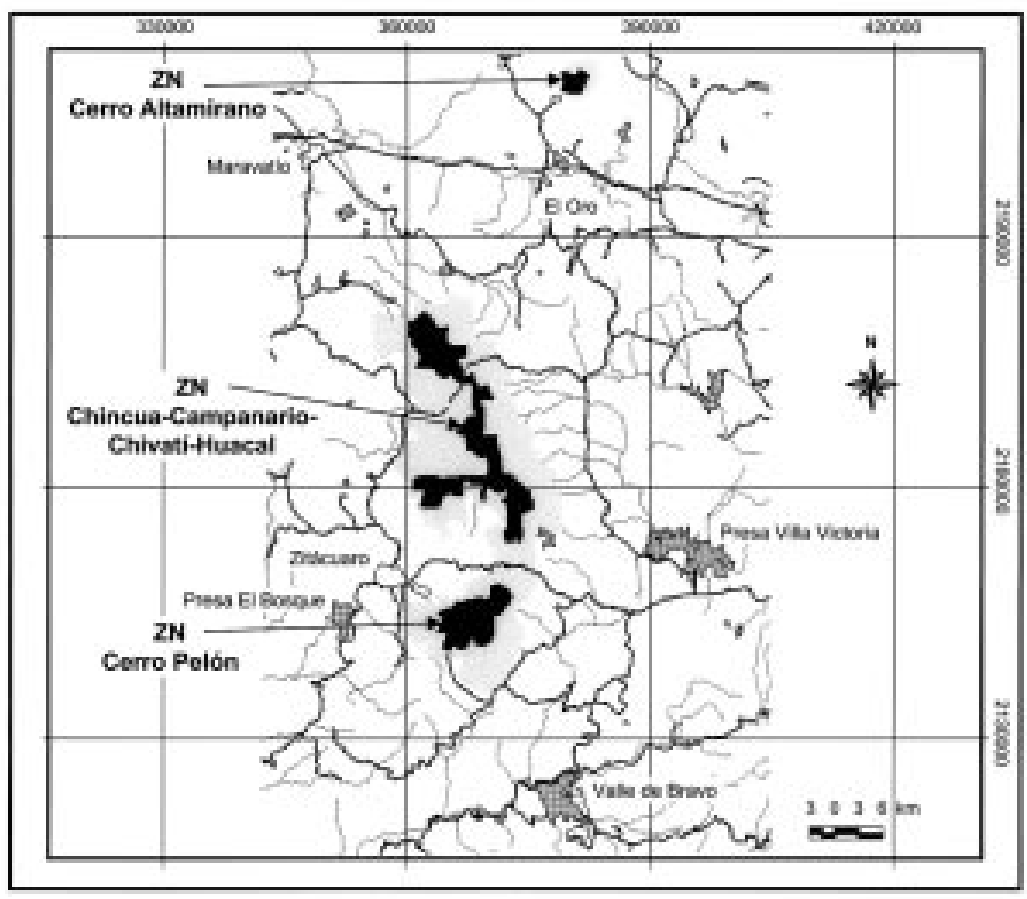

Figura 1. Localización de la Reserva de la Biosfera Mariposa Monarca en los estados de México y Michoacán. 
estado de México (figura 1). Actualmente la reserva tiene una superficie de 56,259 ha, dividida en tres zonas núcleo (13,551 ha): Cerro Altamirano, el corredor ChincuaCampanario-Chivatí-Huacal, y Cerro Pelón y una zona de amortiguamiento (Diario Oficial de la Federación, 2000a).

La región pertenece a la provincia morfotectónica denominada como Faja Volcánica Transmexicana, caracterizada por la presencia de cuerpos volcánicos originados entre el Cenozoico Medio y el Tardío, constituidos básicamente por rocas extrusivas de basalto, riolitas y tobas (Ferrusquía-Villafranca, 1993). De la superficie total, $77 \%$ está compuesto por sierras y lomeríos y el resto por pequeños valles intermontanos y llanuras; la altitud varía de 2,400 a 3,600 m s.n.m. (SEMARNAT, 2001). Los suelos predominantes en el área son andosoles (cerca de $43 \%$ ), aunque en menor extensión también se han registrado acrisoles, planosoles, feozem, litosoles, luvisoles, cambisoles, regosoles y vertisoles, todos ellos derivados de cenizas volcánicas (SEMARNAP, 1998). La zona forma parte de dos regiones hidrológicas: la del río Lerma-Santiago hacia el norte y la del río Balsas en dirección sur (GómezPompa y Dirzo, 1995).

En el área se registran temperaturas medias anuales de 8 a $22^{\circ} \mathrm{C}$ y precipitaciones anuales totales desde 700 hasta 1,250 $\mathrm{mm}$. Se presentan siete subtipos climáticos (SEMARNAP, 1998): (1) (A) $\mathrm{Cw}_{0}$, el más seco de los semitemplados subhúmedos; (2) (A) $\mathrm{Cw}_{1}$, semitemplado subhúmedo, que es intermedio en humedad; (3) (A) $\mathrm{Cw}_{2}$, el más húmedo de los semitemplados subhúmedos; (4) $\mathrm{Cw}_{1}$, templado subhúmedo, intermedio en humedad; (5) $\mathrm{Cw}_{2}$, el más húmedo de los templados subhúmedos; (6) $\mathrm{C}(\mathrm{E}) \mathrm{w}_{2}$, el más húmedo de los semifríos subhúmedos; y (7) C(E)m, semifrío húmedo.

De acuerdo con Rzedowski (1978), la RBMM se localiza en la provincia florística de las Serranías Meridionales, en la región Mesoamericana de Montaña. Este autor indica que los géneros Quercus (Fagaceae), Salvia (Lamiaceae), Eupatorium, Senecio, Stevia (Asteraceae) y Muhlenbergia (Poaceae) presentan en esta provincia un importante centro de diversificación. De acuerdo a Gómez-Pompa y Dirzo (1995), la reserva presenta los siguientes tipos de vegetación: bosque de pino, bosque de encino, matorral secundario de Juniperus, bosque de Cupressus, comunidad de AbiesCupressus, comunidad de Abies-Pinus, comunidad de Quercus-Pinus y comunidad de Pinus-Abies. Se pueden consultar descripciones de las comunidades vegetales presentes en la reserva en Espejo-Serna et al. (1992), SotoNúñez y Vázquez-García (1993), Mejía-Mendoza (1996), Pinto-León (2000) y Genchi (2002).

\section{Materiales y métodos}

Se realizaron recorridos de campo para recolectar muestras botánicas de febrero de 2000 a enero de 2001, las cuales se hicieron principalmente en las zonas núcleo de la RBMM.
Para la determinación del material botánico y la actualización taxonómica de los nombres de las especies se usaron diversas obras florísticas (p.ej. Zanoni, 1982; McVaugh, 1984, 1987; Bello-González y Labat, 1987; Carranza-González, 1992; Martínez, 1992; González-Rivera, 1993; Nixon, 1993; Styles , 1993; Huft, 1994; Zamudio y Carranza, 1994; Fernández, 1996; Espinosa-García y Sarukhán, 1997; Espejo-Serna y López-Ferrari, 1998; Rzedowski y Calderón de Rzedowski, 1998; Calderón de Rzedowski y Rzedowski; 2001; MéndezLarios y Villaseñor, 2001). La determinación de los ejemplares recolectados fue cotejada con muestras depositadas en el herbario del Instituto de Ecología, A.C., del Centro Regional del Bajío (IEB); diversos grupos fueron revisados por especialistas (p.ej. Asteraceae y Poaceae). Un juego completo de los ejemplares recolectados se depositó en el herbario IEB. Asimismo, se entregó material botánico a los herbarios del Instituto de Biología de la Universidad Nacional Autónoma de México (MEXU) y de la Universidad Michoacana de San Nicolás de Hidalgo (EBUM).

Para las especies citadas para la reserva por Ibarra (1983), Espejo-Serna et al. (1992), Mejía-Mendoza (1996), PintoLeón (2000), Farfán-Heredia (2001) y SEMARNAT (2001), se buscaron especímenes en los herbarios IEB, EBUM y MEXU. La revisión bibliográfica permitió detectar que numerosos nombres de las especies citados en estos trabajos son considerados ahora como sinónimos; estos conforman una lista de más de 400 nombres que pueden ser consultados por solicitud directa al autor para la correspondencia (GIM). La lista florística del presente trabajo incluye únicamente las especies que cuentan con ejemplares de herbario, los cuales certifican la presencia de dichas especies en la reserva, excepto para Furcraea bedinghausii (Agavaceae) y Psacalium peltatum (Asteraceae), que no se detectaron en estado reproductivo durante el periodo que comprendió el trabajo de campo, por lo que se decidió no colectarlas sin estructuras reproductivas. La lista florística se encuentra arreglada de acuerdo a la clasificación de Cronquist (1981) para las familias de Dicotiledóneas, el de Dahlgren et al. (1985) para las familias de Monocotiledóneas, mientras que para las Pteridofitas se utilizó el sistema seguido por la Flora Mesoamericana (Moran, 1995a, 1995b, 1995c, 1995d y Moran y Yatskievych, 1995). El nombre de los autores de las especies se presenta con base en Villaseñor (2001).

La descripción de los tipos de vegetación se basó en la clasificación de Rzedowski (1978), por lo que se reconocen cuatro principales tipos de vegetación: el bosque de coníferas, el bosque mesófilo de montaña, el bosque de Quercus y los pastizales o zacatonales antropógenos.

\section{Resultados}

Flora. La lista florística consta de 423 especies que incluyen 32 taxa infraespecíficos, agrupadas en 244 géneros y 87 familias (apéndice 1). La clase Magnoliopsida conforma el 
Cuadro 1. Riqueza de los grupos de plantas vasculares en las zonas núcleo de la Reserva de la Biosfera Mariposa Monarca.

\begin{tabular}{lcccc}
\hline Taxa & Familias & Géneros & Especies & $\begin{array}{c}\text { Especies } \\
\text { endémicas }\end{array}$ \\
\hline Pteridophyta & 6 & 10 & 12 & - \\
$\begin{array}{l}\text { Coniferophyta } \\
\text { Magnoliophyta }\end{array}$ & 2 & 4 & 8 & 2 \\
$\quad$ Magnoliopsida & 66 & 194 & 356 & 120 \\
$\quad$ Liliopsida & 13 & 36 & 47 & 13 \\
Total & 87 & 244 & 423 & 135 \\
\hline
\end{tabular}

Cuadro 2. Familias y géneros con mayor número de especies en la zona de estudio.

\begin{tabular}{lclc}
\hline Familia & $\begin{array}{c}\text { Número de } \\
\text { especies }\end{array}$ & Género & $\begin{array}{c}\text { Número de } \\
\text { especies }\end{array}$ \\
\hline Asteraceae & 103 & Salvia (Lamiaceae) & 13 \\
Lamiaceae & 21 & Stevia (Asteraceae) & 8 \\
Fabaceae & 17 & Ageratina (Asteraceae) & 7 \\
Scrophulariaceae & 17 & Geranium (Geraniaceae) & 6 \\
Solanaceae & 14 & Senecio (Asteraceae) & 6 \\
Rosaceae & 13 & Sisyrinchium (Iridaceae) & 6 \\
Poaceae & 12 & Solanum (Solanaceae) & 6 \\
Apiaceae & 11 & Eryngium (Apiaceae) & 5 \\
Brassicaceae & 10 & Quercus (Fagaceae) & 5 \\
Caryophyllaceae & 10 & Tagetes (Asteraceae) & 5
\end{tabular}

grupo con mayor número de miembros a nivel de familias, géneros y especies (cuadro 1). El cuadro 2 muestra las familias y géneros con más especies presentes en las zonas núcleo de la RBMM. A nivel de familias, Asteraceae resalta del resto de los taxa, incluyendo $24.3 \%$ del total de especies encontradas, siguiendo en orden de importancia Lamiaceae, Fabaceae y Scrophulariaceae. Las diez familias con mayor número de especies representan $53.9 \%$ de la flora total, mientras que 35 cuentan con una especie. El género Salvia (Lamiaceae), con 13 especies, resultó ser el más importante en cuanto a su diversidad; entre los diez géneros más diversos, cuatro pertenecen a Asteraceae (cuadro 2). Un total de 135 especies (31.9\%), entre las cuales están incluidos 13 taxa infraespecíficos, son endémicas de México. El mayor número de especies de esta categoría se encuentra en Magnoliopsida y ninguna se ha detectado hasta el momento para Pteridophyta (cuadro 2). Cerca de la mitad de las familias $(41,47.1 \%$ ) cuentan con especies endémicas entre sus miembros, aunque nuevamente Asteraceae destaca al respecto, con 40 especies (29.6\% ), seguida por Lamiaceae con 11 especies.

En cuanto a las formas de crecimiento, la flora de la reserva está dominada por las hierbas, con 326 especies (77.1\%), con una menor proporción de arbustos $(51,12.1 \%)$, árboles $(33,7.8 \%)$, parásitas (seis arbustivas y una herbácea, $1.7 \%)$ y hierbas epífitas $(6,1.4 \%)$. Ocho especies presentaron el hábito trepador: cinco hierbas (Galium aschenbornii, $G$. mexicanum, Smilax moranensis, Solanum appendiculatum y Valeriana clematitis) y tres leñosas (Archibaccharis hirtella, Clematis dioica y Cyclanthera ribiflora). La forma de crecimiento predominante entre las especies endémicas es la herbácea (97 especies, $71.8 \%$ ), seguida de la arbustiva y la arbórea, con $15.5 \%$ y $7.4 \%$, respectivamente.

En la reserva existen cinco especies sujetas a protección especial (Diario Oficial de la Federación, 2000b): Cupressus lusitanica (Cupressaceae), Comarostaphylis discolor (Ericaceae), Dahlia scapigera (Asteraceae), Gentiana spathacea (Gentianaceae) y Juniperus monticola (Cupressaceae), mientras que Furcraea bedinghausii está catalogada como una especie amenazada.

Vegetación. El tipo de vegetación predominante en las zonas núcleo de la reserva es el bosque de coníferas, el cual se establece aproximadamente entre 2,400 y 3,300 m de altitud y puede entremezclarse con otros tipos de vegetación, como por ejemplo el bosque de Quercus. El estrato arbóreo puede estar compuesto por diferentes combinaciones de árboles de 20 a $40 \mathrm{~m}$ de altura, entre los que destacan Abies religiosa, que es una especie que puede llegar a formar comunidades casi monoespecíficas en este estrato, Arbutus xalapensis, Cupressus lusitanica, Pinus hartwegii, P. pseudostrobus y Quercus laurina. El estrato arbóreo medio y bajo lo constituyen árboles de 10 a 25 m, como Arbutus xalapensis, Buddleia parviflora, B. cordata, Comarostaphylis discolor, Garrya laurifolia, Quercus laurina, Salix paradoxa y Sambucus nigra var. canadensis. El estrato arbustivo mide hasta $5 \mathrm{~m}$ y está dominado por Acaena elongata, Ageratina glabrata, A. mairetiana, Cestrum thyrsoideum, Roldana albonervia, $R$. angulifolia y Satureja macrostema. La presencia de Baccharis conferta y Lupinus montanus se favorece cuando ocurre un fuerte disturbio en el bosque, sobre todo incendios. Las hierbas son abundantes, predominando individuos pertenecientes a especies de compuestas (p.ej. Cirsium ehrenbergii, Dahlia scapigera, Erigeron galeottii, Roldana lineolata, Piqueria pilosa, Senecio callosus, S. toluccanus, Sigesbeckia jorullensis, y Stevia jorullensis) y de otras familias como Alchemilla procumbens, Castilleja tenuiflora, Cyperus seslerioides, Echeveria secunda, Geranium lilacinum, G. potentillaefolium, Govenia liliacea, Malaxis soulei, Oxalis alpina, Poa апnиа, Polypodium madrense, Salvia elegans, S. fulgens, S. prunelloides, Sedum minimum, Smilax moranensis, Valeriana pulchella, Vulpia myurus y Weldenia candida. En algunas partes del Cerro 
Pelón existen manchones de un pinar dominado casi exclusivamente por Pinus leiophylla. El bosque de coníferas presenta una conspicua variante regional: el matorral de Juniperus. Esta comunidad parece ser una comunidad sucesional del bosque de Abies y presenta una fisonomía muy característica, ya que carece de un estrato arbóreo, sobresaliendo el estrato arbustivo con una altura de hasta 2.5 $\mathrm{m}$, compuesto casi en su totalidad por Juniperus monticola $\mathrm{f}$. monticola, que puede estar acompañada infrecuentemente por Ribes affine y en condiciones de disturbio por Baccharis conferta. En el estrato herbáceo se encuentran Astragalus oxyrrhynchus, Daucus montanus, Gentianella amarella subsp. hartwegii, G. amarella subsp. mexicana, Hypericum philonotis y Juncus ebracteatus. Otra comunidad aparentemente sucesional del bosque de coníferas es el matorral de Baccharis heterophylla, que puede alcanzar 2.5 $\mathrm{m}$ de alto. Aunque es necesario realizar estudios para determinar su dinámica sucesional, este tipo de agrupación es considerado en este trabajo como una etapa sucesional del bosque de oyamel debido a que: (1) se trata de comunidades prácticamente contiguas, y (2) la presencia de individuos juveniles de Abies religiosa que están creciendo debajo de los individuos adultos de Baccharis, lo que sugiere que éstas se desempeñan como plantas nodrizas de los oyameles. Entre los componentes herbáceos se pueden mencionar a Geranium seemannii, Eleocharis acicularis, Eriocaulon benthamii, Hypoxis mexicana, Ranunculus dichotomus, Sisyrinchium schaffneri, Veronica peregrina subsp. xalapensis y $V$. serpyllifolia.

El bosque mesófilo de montaña se encuentra en pequeñas áreas y cañadas en Cerro Pelón y Chincua, ocupando un estrecho intervalo altitudinal (2,500 a 2,700 m). Las especies arbóreas dominantes son Abies religiosa, Alnus jorullensis subsp. jorullensis, Clethra mexicana, Comarostaphylis longifolia, Holodiscus pachydiscus, Oreopanax xalapensis, Quercus laurina, Salix paradoxa, Ternstroemia lineata y Viburnum acutifolium. El estrato arbustivo está representado por Bocconia frutescens, Roldana angulifolia, R. barbajohannis, Satureja macrostema, Verbesina klattii y V. oncophora. Las hierbas más notables son Adiantum andicola, Asplenium monanthes, Dahlia rudis, Drymaria malachioides, Heuchera orizabensis, Lobelia laxiflora var. laxiflora, Lupinus montanus, Nama prostratum, Sabazia humilis, Salvia mexicana, Senecio callosus y Valeriana clematitis.

Entre 2,500 y 2,900 m s.n.m. se encuentra el bosque de Quercus, generalmente en áreas muy pequeñas, mezclándose con especies de coníferas, principalmente con especies de pinos. Las mayores extensiones están en las zonas núcleo Cerro Altamirano y en menor medida en la de Cerro Pelón. El estrato arbóreo alcanza entre 10 y $15 \mathrm{~m}$, destacando la presencia de Quercus laurina, Q. obtusata, Q. rugosa, Arbutus xalapensis y, en menor abundancia, de Clethra mexicana. El estrato arbustivo, de 0.5 a $2 \mathrm{~m}$, tiene entre las especies más conspicuas a Ageratina areolaris, Arctostaphylos pungens, A. rupestris y Bouvardia longiflora. Las plantas herbáceas de este bosque son abundantes, sobresaliendo Bouvardia ternifolia, Cosmos scabiosoides, Dahlia coccinea, Gentiana spathacea, Helianthemum glomeratum, Lamouroxia multifida, Loeselia mexicana, Penstemon roseus, Pinguicula moranesis, Piqueria trinervia, Salvia mexicana y $S$. patens. Las epífitas están representadas por las bromeliáceas Tillandsia andrieuxii, T. erubescens y $T$. macdougallii. Entre las plantas parásitas se encuentran Cladocolea diversifolia y Phoradendron schumanni.

Finalmente, se presentan los zacatales antropógenos, caracterizados por la dominancia en cobertura de gramíneas, aunque entremezclados con los pastos es posible encontrar una notable riqueza de especies de plantas herbáceas pertenecientes a otras familias. Esta comunidad se localiza a lo largo del gradiente altitudinal encontrado en el área y está frecuentemente asociada a disturbios producto de actividades humanas, principalmente de tala de comunidades boscosas y ganadería. Entre las plantas más conspicuas están las especies de compuestas Helenium scorzonerifolium, Packera sanguisorbae y Senecio toluccanus, de hasta $1 \mathrm{~m}$ de alto cuando llegan a su fase reproductiva, las cuales llegan a formar manchones muy notorios en sitios con suelos de drenaje deficiente. Las especies mencionadas suelen encontrarse mezcladas con Chromolepis heterophylla, Eryngium subacaule, Gentianella amarella subsp. mexicana, Mimulus glabratus, Pedicularis mexicana, Ranunculus dichotomus, Senecio helodes y Sisyrinchium conzattii. En los sitios menos húmedos se encuentran principalmente Achillea millefolium, Acmella oppositifolia, Bromus exaltatus, Echeandia flavescens, Halenia brevicornis, Hedyotis pygmaea, Penstemon gentianoides, Piptochaetium fimbriatum, Setaria parviflora y Stachys radicans.

Con respecto al número de especies por tipo de vegetación, el más diverso es el bosque de coníferas con 322 especies (76.1\% del total de especies), siguiendo en orden de importancia el bosque de Quercus (82). Estas comunidades vegetales son también las más importantes con respecto a las especies restringidas a un solo tipo de vegetación, con 250 y 48 especies, respectivamente (figura 2).

\section{Discusión}

La presente contribución incluye a las especies recolectadas en las zonas núcleo de la RBMM, aunque un pequeño porcentaje fue recolectado exclusivamente en una comunidad Mazahua ubicada en la zona de amortiguamiento (FarfánHeredia, 2001). La revisión de estudios de flora y vegetación del área de estudio realizada por Cornejo-Tenorio (2002) generó una lista de 145 especies adicionales a las citadas en el presente estudio. Para estas especies no fue posible localizar ejemplares de referencia en los herbarios EBUM, IEB, y MEXU, por lo que su presencia en el área es por el momento incierta. A pesar de que no se cuenta con información 


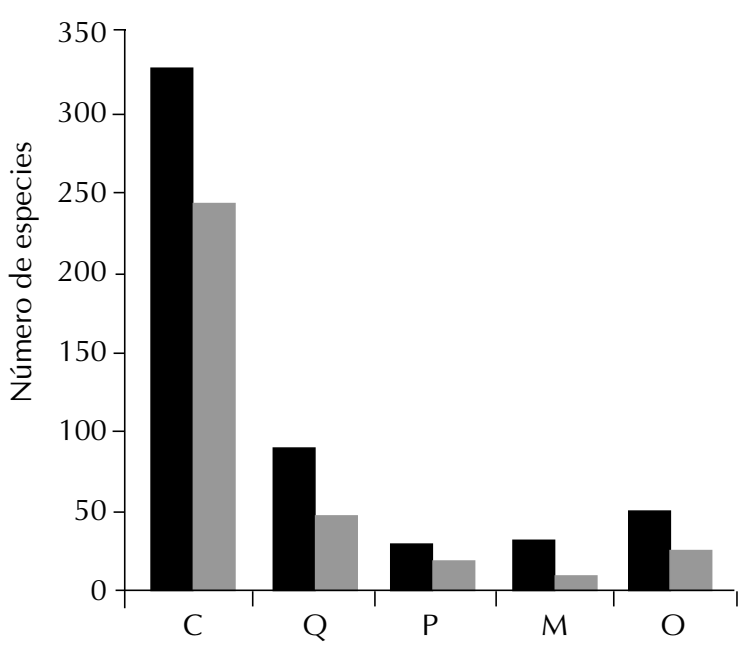

Figura 2. Número de especies de plantas vasculares (barras negras) para los diferentes tipos de vegetación presentes en la Reserva de la Biosfera Mariposa Monarca. Las barras grises indican el número de especies restringidas a cada comunidad vegetal. Tipos de vegetación: $\mathrm{C}$ (bosque de coníferas), $\mathrm{M}$ (bosque mesófilo de montaña), Q (bosque de Quercus), P (pastizal antropogénico), O (otros).

cuantitativa sobre las afinidades florísticas entre las áreas núcleo, las observaciones de campo realizadas en este estudio sugieren que su composición florística es muy similar, particularmente en lo que se refiere a las especies más abundantes. Los datos aportados por Mejía-Mendoza (1996) consolidan esta afirmación, ya que de las 226 especies que registró en los santuarios y que ahora corresponden a las zonas núcleo de la reserva, sólo $15.5 \%$ fueron encontradas en uno de los sitios, mientras que $31 \%$ se registró en todos los santuarios.

La familia Asteraceae, con 103 especies, es la mejor representada en la reserva, seguida en una menor proporción por Lamiaceae, Fabaceae, Scrophulariaceae, Solanaceae y Rosaceae. Es notable la preponderancia de Asteraceae con cerca de $25 \%$ de las especies, así como el hecho de que las seis familias mencionadas contengan $43.7 \%$ del total de la flora del área. Este patrón de predominio florístico ha sido también encontrado en trabajos previos realizados en tipos de vegetación similares a los del área de estudio, por ejemplo, el Parque Nacional Lagunas de Zempoala (Bonilla-Barbosa y Viana-Lases, 1997), el área de San Juan Nuevo Parangaricutiro (Medina-García et al., 2000) y el área del Valle de México (Calderón de Rzedowski y Rzedowski, 2001). Con respecto a los géneros más diversos, Vázquez et al. (1995) y Medina-García et al. (2000) destacan la importancia al respecto de Eupatorium s.l. y Salvia, lo que coincide con los datos del presente estudio, si bien el primero de éstos ha sido segregado en varios géneros, de los cuales su representante más importante en la RBMM es el género Ageratina (cuadro 2).
Del total de las especies registradas para las zonas núcleo de la reserva, $31.9 \%$ son endémicas de México y, de éstas, una tercera parte pertenece a la familia Asteraceae. Este alto valor de endemismo es consistente con evaluaciones previas de la flora de las regiones montañosas de México, ya que Rzedowski (1991a) estima que la proporción de especies endémicas del país para los bosques de coníferas y de encino equivale a $70 \%$ y para el bosque mesófilo de montaña, a $30 \%$. Patrones similares han sido encontrados para algunos grupos taxonómicos. Por ejemplo, alrededor de $60 \%$ de las especies de Asteraceae son endémicas del país (Turner y Nesom, 1993; Villaseñor, 1993). Ramamoorthy y Elliott (1998) indican que al menos $77 \%$ de las especies de Lamiaceae no sobrepasan los límites políticos de México y se les encuentra principalmente en la Sierra Madre Occidental, Sierra Madre Oriental, Eje Volcánico Transversal y las montañas de Chiapas y Oaxaca. La relevancia de las porciones montañosas de México respecto al endemismo de la flora mexicana es ratificada por Delgadillo et al. (2003), quienes indicaron que el Eje Volcánico Transversal es un área extremadamente relevante por albergar un alto porcentaje de especies mexicanas de compuestas, gramíneas y musgos. A nivel de género, Quercus y Pinus son citados frecuentemente como elementos típicos de la flora templada mexicana, con un endemismo que oscila entre 60 y $66 \%$ para los encinos (González-Rivera, 1993; Nixon, 1993) y alrededor de 53\% para los pinos (Styles, 1993). Rzedowski (1991b) propone que una de las causas más importantes para explicar el endemismo de las fanerógamas mexicanas debe de ser el desempeño como "islas ecológicas" de las porciones montañosas con clima templado y semihúmedo, lo cual se combina con la gran diversidad ambiental y la compleja historia geológica de México. En cuanto a las formas de crecimiento, las hierbas son las más numerosas $(77.1 \%)$, seguidas por los árboles y los arbustos. Una tendencia similar fue encontrada por Medina-García et al. (2000), incluyendo un bajo porcentaje de epífitas, que para la RBMM sólo estuvo representada por seis especies.

Por otro lado, Velázquez et al. (2000) afirman que la clasificación de los tipos de vegetación templada en México enfatiza la composición genérica de las especies del dosel, sin describir con precisión la gran variación en composición y estructura de sus estratos herbáceo y arbustivo. Entre las principales comunidades templadas y los géneros más característicos que reconocen estos autores en su trabajo se encontró una coincidencia con algunos taxa para el bosque de coníferas presente en la reserva (Arbutus, Buddleia, Roldana y Stevia).

Es importante que en el futuro se continúe con la exploración botánica de la reserva, particularmente en su extensa zona de amortiguamiento, a fin de contar con una lista completa y actualizada de su flora vascular. Lo anterior debe acoplarse con el desarrollo de estudios poblacionales que permitan determinar los cambios espaciales y temporales 
para distintas especies (p.ej., las más abundantes para cada tipo de vegetación), cuantificando la influencia del severo disturbio provocado por las actividades humanas, el cual se manifiesta principalmente por actividades de ganadería y tala clandestina, inclusive en las zonas núcleo. La realización de estudios de vegetación para determinar la estructura, la composición y la dinámica sucesional deben ser una prioridad de investigación en el área, particularmente en las zonas núcleo Cerro Altamirano y Cerro Pelón, ya que para ellas la información al respecto es escasa o nula, además de que sus comunidades vegetales exhiben un grado de disturbio comparativamente menor que el que se observa en la zona núcleo de Chincua-Campanario-Chivatí-Huacal.

Los resultados que se presentan en este estudio muestran el papel fundamental que desempeña la RBMM como área de conservación de la flora mexicana, no sólo por la riqueza de sus elementos sino también por el importante porcentaje de especies endémicas mexicanas y amenazadas que protege. Se espera que la información florística proporcionada por la presente contribución pueda ser útil al plan de manejo de la reserva y que fomente estudios similares en otras áreas protegidas de México, entre las cuales existen muy pocos ejemplos que documenten la flora que protegen.

\section{Agradecimientos}

Al personal de la RBMM, especialmente a Eligio García Serrano, por su disposición a colaborar en el trabajo de campo. Se agradece también la colaboración en la determinación de especies a Francisco Santana Michel (Cyperaceae, Poaceae), Sergio Zamudio Ruiz (Lentibulariaceae) y Socorro González Elizondo (Ericaceae). Esta investigación contó con apoyo financiero otorgado por el SIMORELOS/CONACYT (proyecto 19990306007). La autora principal gozó de una beca de licenciatura otorgada por la Dirección General de Apoyo del Personal Académico de la Universidad Nacional Autónoma de México (proyecto INI101900). El contenido y estructura del presente trabajo fue mejorado por las atinadas sugerencias de Socorro González Elizondo, Luis Gerardo Hernández Sandoval, Emmanuel Pérez Cálix y Jorge A. Meave. Se reconoce la ayuda de Fernando A. Roseta V., Vicente Salinas Melgoza y Marco Antonio Romero Romero para la elaboración de la figura 1 .

\section{Literatura citada}

Alonso A. y Alonso L.E. 1999. Scientific research and social investigation priorities for the Monarch Butterfly Special Biosphere Reserve: recommendations. En: Hoth J., Merino L., Oberhauser K., Pisanty I., Price S. y Wilkinson T. Comps. Reunión de América del Norte sobre la Mariposa Monarca, 1997, pp. 183-194, Comisión para la Cooperación Ambiental, Montreal, Quebec.

Bernal-Hernández M.A. y Camacho-Vera A.D. 1999. Análisis crítico del manejo de la Reserva Especial de la Biosfera Mariposa Monarca. En: Hoth J., Merino L., Oberhauser K., Pisanty I., Price S. y Wilkinson T. Comps. Reunión de América del Norte Sobre la Mariposa Monarca, 1997, pp. 315-322, Comisión para la Cooperación Ambiental, Montreal, Quebec.

Bello-González M.A. y Labat J.N. 1987. Los Encinos (Quercus) del Estado de Michoacán, México. Secretaría de Agricultura y Recursos Hidráulicos, CEMCA, México, D.F.

Bonilla-Barbosa J.R. y Viana-Lases J.A. 1997. Listados Florísticos de México XIV. Parque Nacional Lagunas de Zempoala. Instituto de Biología, Universidad Nacional Autónoma de México, México, D.F.

Broker L.P. 1999. Biological necessities for monarch butterfly overwintering in relation to the oyamel forest ecosystem in Mexico. En: Hoth J., Merino L., Oberhauser K., Pisanty I., Price S. y Wilkinson T. Comps. Reunión de América del Norte Sobre la Mariposa Monarca, 1997, pp. 11-28, Comisión para la Cooperación Ambiental, Montreal, Quebec.

Calderón de Rzedowski G. y Rzedowski J. 2001. Eds. Flora Fanerogámica del Valle de México. $2^{\mathrm{a}}$ ed. Instituto de Ecología, A.C., Centro Regional del Bajío, Comisión Nacional para el Conocimiento y Uso de la Biodiversidad, Pátzcuaro, Michoacán.

Carranza-González E. 1992. Cornaceae. Flora del Bajío y de Regiones Adyacentes 8:1-11.

Cornejo-Tenorio G. 2002. Fitogeografía de la Reserva de la Biosfera Mariposa Monarca, Michoacán, México. Tesis Profesional, Universidad Michoacana de San Nicolás de Hidalgo, Morelia, Michoacán, 123 pp.

Cox C.B. 2001. The biogeographic regions reconsidered. Journal of Biogeography 28:511-523.

Cronquist A. 1981. An Integrated System of Classification of Flowering Plants. Columbia University Press, Nueva York.

Dahlgren R.M.T., Clifford H.T. y Yeo P.F. 1985. The Families of the Monocotyledons. Springer-Verlag, Berlín, Heidelberg.

Delgadillo C. 1993. Diversity in the Mexican bryoflora. En: Ramamoorthy T.P., Bye R., Lot A. y Fa J. Eds. Biological Diversity of Mexico: Origins and Distribution, pp. 365-377, Oxford University Press, Nueva York.

Delgadillo C., Villaseñor J.L. y Dávila P. 2003. Endemism in the Mexican flora: a comparative study in three plant groups. Annals of the Missouri Botanical Garden 90:25-34.

Diario Oficial de la Federación. 2000a. Decreto por el que se declara área natural protegida, con el carácter de Reserva de la Biosfera, la región denominada Mariposa Monarca, ubicada en los municipios de Temascalcingo, San Felipe del Progreso, Donato Guerra y Villa de Allende en el Estado de México, así como en los municipios de Contepec, Senguio, Angangueo, Ocampo, Zitácuaro y Aporo en el Estado de Michoacán, con una superficie total de 56,259-05-07.275 hectáreas, Primera sección. Secretaría del Medio Ambiente, Recursos Naturales y Pesca, pp. 5-28, 10 de noviembre de 2000.

Diario Oficial de la Federación. 2000b. Proyecto de Norma Oficial Mexicana PROY-NOM-059-ECOL-2000, Protección ambiental - Especies de flora y fauna silvestres de México - Categorías de riesgo y especificaciones para su inclusión, exclusión o cambio - Lista de especies en riesgo. Secretaría del Medio Ambiente, Recursos Naturales y Pesca. México, D.F.

Escalante-Pliego P., Navarro-Sigüenza A.G. y Townsed-Peterson A. 1993. A geographic, ecological, and historical analysis of land bird diversity in Mexico. En: Ramamoorthy T.P., Bye R., Lot A. 
y Fa J. Eds. Biological Diversity of Mexico: Origins and Distribution, pp. 281-307, Oxford University Press, Nueva York.

Espejo-Serna A., Brunhuber-Menéndez J.L., Segura-Warnholtz G. e Ibarra-Caballero J. 1992. La vegetación de la zona de hibernación de la mariposa monarca (Danaus plexippus L.) en la Sierra Chincua. Tulane Studies in Zoology and Botany. Supplementary Publication 1:79-99.

Espejo-Serna A. y López-Ferrari A.R. 1998. Iridaceae. Flora de Veracruz 105:1-58.

Espinosa-García F.J. y Sarukhán J. 1997. Manual de Malezas del Valle de México. Claves, Descripciones e Ilustraciones. Ediciones Científicas Universitarias, Universidad Nacional Autónoma de México, Fondo de Cultura Económica, México, D.F.

Fa J.E. y Morales L.M. 1993. Patterns of mammalian diversity in Mexico. En: Ramamoorthy T.P., Bye R., Lot A. y Fa J. Eds. Biological Diversity of Mexico: Origins and Distribution, pp. 319-361, Oxford University Press, Nueva York.

Farfán-Heredia B. 2001. Aspectos ecológicos y etnobotánicos de los recursos vegetales de la comunidad Mazahua Francisco Serrato, municipio de Zitácuaro, Michoacán, México. Tesis Profesional, Facultad de Biología, Universidad Michoacana de San Nicolás de Hidalgo, Morelia, Michoacán,137 pp.

Fernández N.R. 1996. Rhamnaceae. Flora del Bajío y de Regiones Adyacentes 43:1-69.

Ferrusquía-Villafranca I. 1993. Geology of Mexico: a synopsis. En: Ramamoorthy T.P., Bye R. Lot A. y Fa J. Eds. Biological Diversity of Mexico: Origins and Distribution, pp. 54-60, Oxford University Press, Nueva York.

Flores-Villela O. 1993. Herpetofauna of Mexico: distribution and endemism. En: Ramamoorthy T.P., Bye R., Lot A. y Fa J. Eds. Biological Diversity of Mexico: Origins and Distribution, pp. 253-280, Oxford University Press, Nueva York.

Genchi L. 2002. The vegetation of Sierra de Chincua, Monarch Butterfly Special Biosphere Reserve, Michoacán, México. Tesis de Maestría, Wageningen University, Wageningen, Holanda, 128 pp.

Gómez-Pompa A. y Dirzo R. 1995. Reservas de la Biosfera y otras Áreas Naturales Protegidas de México. SEMARNAP, INE y CONABIO, México, D.F.

González-Rivera R. 1993. La diversidad de los encinos mexicanos. Revista de la Sociedad Mexicana de Historia Natural 44:125142.

Huft M.J. 1994. Smilacaceae. En: Davidse G., Sousa S.M. y Chater A.O. Eds. Flora Mesoamericana: Alismataceae a Cyperaceae, Vol. 6, pp. 20-25, Instituto de Biología, Universidad Nacional Autónoma de México, Missouri Botanical Garden, The Natural History Museum (London), México, D.F.

Ibarra G. 1983. Comunidades vegetales del Cerro El Cacique, ubicado en el Eje Neovolcánico; Zitácuaro, Michoacán. Tesis Profesional, Facultad de Ciencias, Universidad Nacional Autónoma de México, México, D.F., 105 pp.

Martínez M. 1992. Los Pinos Mexicanos. $3^{\mathrm{a}}$ ed. Ediciones Botas, México, D.F.

McVaugh R. 1984. Compositae. En: Anderson W.R. Ed. Flora NovoGaliciana. A descriptive account of the vascular plants of Western Mexico, Vol. 12, pp. 1-1157, The University of Michigan Press, Ann Arbor.

McVaugh R. 1987. Leguminosae. En: Anderson W.R. Ed. Flora Novo-Galiciana. A descriptive account of the vascular plants of Western Mexico, Vol. 5, pp. 1-786, The University of Michigan Press, Ann Arbor.
Medina-García C., Guevara-Féfer F., Martínez-Rodríguez M.A., Silva-Sáenz P. y Chávez-Carbajal M.A. 2000. Estudio florístico en el área de la comunidad indígena de Nuevo San Juan Parangaricutiro, Michoacán, México. Acta Botanica Mexicana 52:5-41.

Melo-Gallegos C. y López-García J. 1989. Contribución geográfica al Programa Integral de Desarrollo Mariposa Monarca. Boletín del Instituto de Geografía, UNAM 19:9-26.

Mejía-Mendoza M.M. 1996. Caracterización de las colonias de la Mariposa Monarca (Danaus plexippus L.) en la Reserva Especial de la Biosfera en el Estado de Michoacán, México. Tesis Profesional, Facultad de Biología, Universidad Michoacana de San Nicolás de Hidalgo, Morelia, Michoacán, 133 pp.

Méndez-Larios I. y Villaseñor J.L. 2001. La familia Scrophulariaceae en México: diversidad y distribución. Boletín de la Sociedad Botánica de México 69:101-121.

Merino L. 1999. Reserva Especial de la Biosfera Mariposa Monarca: problemática general de la región. En: Hoth J., Merino L., Oberhauser K., Pisanty I., Price S. y Wilkinson T. Comps. Reunión de América del Norte Sobre la Mariposa Monarca, 1997, pp. 239-248, Comisión para la Cooperación Ambiental, Montreal, Quebec.

Moran R.C. 1995a. Aspleniaceae. En: Davidse G., Sousa S.M. y Chater A.O. Eds. Flora Mesoamericana: Psilotaceae a Salviniaceae, Vol. 1, pp. 290-325, Instituto de Biología, Universidad Nacional Autónoma de México, Missouri Botanical Garden, The Natural History Museum (London), México, D.F.

Moran R.C. 1995b. Dryopteridaceae. En: Davidse G., Sousa S.M. y Chater A.O. Eds. Flora Mesoamericana: Psilotaceae a Salviniaceae, Vol. 1, pp. 210-226, Instituto de Biología, Universidad Nacional Autónoma de México, Missouri Botanical Garden, The Natural History Museum (London), México, D.F.

Moran R.C. 1995c. Polypodiaceae. En: Davidse G., Sousa S.M. y Chater A.O. Eds. Flora Mesoamericana: Psilotaceae a Salviniaceae, Vol. 1, pp. 333-366, Instituto de Biología, Universidad Nacional Autónoma de México, Missouri Botanical Garden, The Natural History Museum (London), México, D.F.

Moran R.C. 1995d. Woodsiaceae. En: Davidse G., Sousa S.M. y Chater A.O. Eds. Flora Mesoamericana: Psilotaceae a Salviniaceae, Vol. 1, pp. 227-247, Instituto de Biología, Universidad Nacional Autónoma de México, Missouri Botanical Garden, The Natural History Museum (London), México, D.F.

Moran R.C. y Yatskievych G. 1995. Pteridaceae. En: Davidse G., Sousa S.M. y Chater A.O. Eds. Flora Mesoamericana: Psilotaceae a Salviniaceae, Vol. 1, pp. 104-145, Instituto de Biología, Universidad Nacional Autónoma de México, Missouri Botanical Garden, The Natural History Museum (London), México, D.F.

Nixon K.C. 1993. The genus Quercus in Mexico. En: Ramamoorthy T.P., Bye R., Lot A. y Fa J. Eds. Biological Diversity of Mexico: Origins and Distribution, pp. 447-458, Oxford University Press, Nueva York.

Pinto-León M.C. 2000. Los Bosques de Abies religiosa (H.B.K.) Cham. et Schl. y sus etapas de sustitución en la Reserva de la Biosfera Mariposa Monarca: un enfoque fitosociológico. Tesis Profesional, Facultad de Ciencias, Universidad Nacional 
Autónoma de México, México, D.F. 96 pp.

Ramamoorthy T.P. y Elliott M. 1998. Lamiaceae de México: diversidad, distribución, endemismo y evolución. En: Ramamorthy T.P., Bye R., Lot A. y Fa J. Comp. Diversidad Biológica de México: Orígenes y Distribución, pp. 501-526, Instituto de Biología, Universidad Nacional Autónoma de México, México, D.F.

Rzedowski J. 1978. Vegetación de México. Limusa, México, D.F.

Rzedowski J. 1991a. Diversidad y orígenes de la flora fanerogámica de México. Acta Botanica Mexicana 14:3-21.

Rzedowski J. 1991b. El endemismo en la flora fanerogámica mexicana: una apreciación analítica preliminar. Acta Botanica Mexicana 15:47-64.

Rzedowski J. y Calderón de Rzedowski G. 1998. Apocynaceae. Flora del Bajío y de Regiones Adyacentes 70:1-64.

SEMARNAP (Secretaría de Medio Ambiente, Recursos Naturales y Pesca). 1998. Ordenamiento ecológico para la región de la Mariposa Monarca. Instituto Nacional de Ecología, Colegio de México, A.C., México.

SEMARNAT (Secretaría del Medio Ambiente y Recursos Naturales). 2001. Programa de Manejo Reserva de la Biosfera Mariposa Monarca. Comisión Nacional de Áreas Naturales Protegidas, México, D.F., 159 p.

Soto-Núñez J.C. y Vázquez-García L. 1993. Vegetation types of Monarch butterfly overwintering habitat in Mexico. En: Malcolm S.B. y Zalucki M.P. Eds. Biology and Conservation of the Monarch Butterfly, pp. 287-293, Natural History Museum of Los Angeles County, Los Angeles, California.
Styles B.A. 1993. Genus Pinus: a Mexican purview. En: Ramamoorthy T.P., Bye R., Lot A. y Fa J. Eds. Biological Diversity of Mexico: Origins and Distribution, pp. 397-420, Oxford University Press, Nueva York.

Turner B.L. y Nesom G.L. 1993. Biogeography, diversity and endangered or threatened status of Mexican Asteraceae. En: Ramamoorthy T.P., Bye R., Lot A. y Fa J. Eds. Biological Diversity of Mexico: Origins and Distribution, pp. 757-777, Oxford University Press, Nueva York.

Vázquez-G. J.A., Cuevas G.R., Cochrane T.S., Iltis H.H., Santana M.F.J. y Guzmán H.L. 1995. Flora de Manantlán. Plantas vasculares de la Reserva de la Biosfera Sierra de Manantlán Jalisco-Colima, México. Sida, Botanical Miscellany 13. Botanical Research Institute of Texas, Inc., Texas.

Villaseñor J.L. 1993. La familia Asteraceae en México. Revista de la Sociedad Mexicana de Historia Natural 44:117-124.

Villaseñor J.L. 2001. Catálogo de Autores de Plantas Vasculares de México. Instituto de Biología, Universidad Nacional Autónoma de México y Comisión Nacional para el Conocimiento y Uso de la Biodiversidad, México, D.F.

Velásquez A., Toledo V.M. y Luna I. 2000. Mexican Temperate Vegetation. En: Barbour M.G. y Billings W.D. Eds. North American Terrestrial Vegetation, pp. 571-592, Cambridge University Press, Nueva York.

Zamudio S. y Carranza E. 1994. Cupressaceae. Flora del Bajío y de Regiones Adyacentes 29:1-21.

Zanoni T.A. 1982. Cupressaceae. Flora de Veracruz, 23:1-15.

Fecha de recepción: 17 de marzo de 2003

Versión corregida: 12 de septiembre de 2003

Aceptado: 3 de octubre de 2003 
Apéndice 1. Lista de plantas vasculares de las zonas núcleo de la Reserva de la Biosfera Mariposa Monarca.

Abreviaturas. Forma de crecimiento (FC): A (árbol), Ar (arbusto), E (epífita), H (hierba), P (parásita). Tipos de vegetación (TV): A (bosque de Abies), C (bosque de coníferas), $\mathbf{M}$ (bosque mesófilo de montaña), $\mathbf{Q}$ (bosque de Quercus), MB (matorral de Baccharis), MJ (matorral de Juniperus), P (Pastizal), R (ruderal), V (vegetación secundaria). Colectores (C): A (Elizabeth Arellano González), AG (Angeles García Guzmán), C (Guadalupe Cornejo Tenorio), E (Esperanza Arriaga Saldaña), EP (Edgar Pérez Negrón), F (Berenice Farfán Heredia), G (Eleazar Carranza González), H (Horalia Díaz Barriga), J (José Carmen Soto Núñez), L (Selene Rangel Luna), M (María Macaria Mejía Mendoza), MC (Mercedes Chávez Moya), MM (Martín Mejía), N (Antonio Negrete Ovando), P (Emmanuel Pérez Calix), R (Jerzy Rzedowski), S (J. Santos Martínez), T (Rafael Torres), X (Xavier Madrigal Sánchez), Z (Sergio Zamudio R.), S/ $\mathbf{C}$ (sin número de colecta). El asterisco $\left(^{*}\right)$ indica las especies endémicas de México.

\begin{tabular}{cccccc}
\hline Taxa & FC & TV & C
\end{tabular}

\section{DIVISIÓN PTERIDOPHYTA}

ASPLENIACEAE

Asplenium castaneum Schltdl. et Cham.

A. monanthes $\mathrm{L}$.

DRYOPTERIDACEAE

Polystichum distans E.Fourn.

POLYPODIACEAE

Pleopeltis mexicana (Fée) Mickel et Beitel

Polypodium madrense J.Sm.

\section{PTERIDACEAE}

Adiantum andicola Liebm.

Cheilanthes chaerophylla (M.Martens et Galeotti) Kunze

Pellaea cordifolia (Sessé et Moc.) A.R.Sm.

P. ternifolia (Cav.) Link

Pteridium feei (W.Schaffn. ex Fée) Maxon

\section{THELYPTERIDACEAE}

Thelypteris pilosa (M.Martens et Galeotti) Crawford

WOODSIACEAE

Cystopteris fragilis (L.) Bernh.

\section{DIVISIÓN CONIFEROPHYTA}

\section{CUPRESSACEAE}

Cupressus lusitanica Mill.

Juniperus deppeana Steud.

J. monticola Martínez f. monticola *

A

A

Ar

PINACEAE

Abies religiosa (Kunth) Schltdl. et Cham.

Pinus hartwegii Lindl.

P. leiophylla Schiede ex Schltdl. et Cham. *

$P$. pseudostrobus Lindl.

\section{DIVISIÓN MAGNOLIOPHYTA}

\section{CLASE MAGNOLIOPSIDA}

\section{AMARANTHACEAE}

Iresine diffusa Humb. et Bonpl. ex Willd.
$\mathrm{H}$

\begin{tabular}{|c|c|c|}
\hline $\mathrm{H}$ & A & EP 268 \\
\hline $\mathrm{H}$ & A, C, M & C 175, F 9 \\
\hline $\mathrm{H}$ & V & F 342 \\
\hline $\mathrm{E}$ & $A, C$ & F 169, N 3 \\
\hline $\mathrm{H}$ & C & C 490, F 20 \\
\hline $\mathrm{H}$ & A, C, M & C $196,468,476$ \\
\hline $\mathrm{H}$ & A, C & C 48, F 200 \\
\hline $\mathrm{H}$ & $\mathrm{Q}$ & C 345 \\
\hline $\mathrm{H}$ & Q & C 346 \\
\hline $\mathrm{H}$ & M & C 197 \\
\hline $\mathrm{H}$ & C & H 3598, S 1953 \\
\hline $\mathrm{H}$ & A & A 11 \\
\hline
\end{tabular}

A, V

Q

A, C

F 108, A 3

R 42256

C 302, X 3313

$\begin{array}{ccc}\text { A } & \text { A } & \text { C 106, P } 269 \\ \text { A } & \text { A } & \text { C } 76 \\ \text { A } & \text { C } & \text { C } 198 \\ \text { A } & \text { A, C } & \text { C } 137\end{array}$




\begin{tabular}{|c|c|c|c|}
\hline Taxa & FC & TV & $\mathrm{C}$ \\
\hline A. rigida J.M.Coult. et Rose & $\mathrm{H}$ & A, C & $\mathrm{F} 42, \mathrm{~A} 8$ \\
\hline $\begin{array}{l}\text { Cyclospermum leptophyllum (Pers.) Sprague ex Britton } \\
\text { et P.Wilson }\end{array}$ & $\mathrm{H}$ & A & E 10 \\
\hline Daucus montanus Humb. et Bonpl. ex Spreng. & $\mathrm{H}$ & A, MJ & C 214, 261 \\
\hline Eryngium alternatum J.M.Coult. et Rose ${ }^{*}$ & $\mathrm{H}$ & A & N9 \\
\hline E. bonplandii F.Delaroche ${ }^{*}$ & $\mathrm{H}$ & $\mathrm{C}$ & C 252,487, R 52770 \\
\hline E. carlinae F.Delaroche & $\mathrm{H}$ & A & C 41 \\
\hline E. columnare Hemsl. & $\mathrm{H}$ & $\mathrm{C}$ & F 24 \\
\hline E. subacaule Cav. & $\mathrm{H}$ & $\mathrm{P}$ & C 84 \\
\hline Prionosciadium thapsoides (DC.) Mathias & $\mathrm{H}$ & A & C 232,300 \\
\hline Tauschia nudicaulis Schltdl. & $\mathrm{H}$ & A & A 12 \\
\hline \multicolumn{4}{|l|}{ APOCYNACEAE } \\
\hline Vinca major L. & $\mathrm{H}$ & V & F 121 \\
\hline \multicolumn{4}{|l|}{ ARALIACEAE } \\
\hline Oreopanax xalapensis (Kunth) Decne. et Planch. & A & A, C & F 357 \\
\hline \multicolumn{4}{|l|}{ ASTERACEAE } \\
\hline Achillea millefolium L. & $\mathrm{H}$ & $M J, P$ & C $294, \mathrm{MS} / \mathrm{N}$ \\
\hline Acmella oppositifolia (Lam.) R.K.Jansen var. oppositifolia & $\mathrm{H}$ & $\mathrm{P}$ & C 82 \\
\hline Ageratina areolaris (DC.) Gage & Ar & Q & C 390, F 299, \\
\hline A. chiapensis (B.L.Rob.) R.M.King et H.Rob. & $\operatorname{Ar}$ & $\mathrm{C}$ & C 147 \\
\hline A. conspicua (Kunth et Bouché) R.M.King et H.Rob. & $\operatorname{Ar}$ & A & C 94 \\
\hline A. glabrata (Kunth) R.M.King et H.Rob. * & $\operatorname{Ar}$ & A & C 136,140 \\
\hline A. mairetiana (DC.) R.M.King et H.Rob. var. mairetiana & Ar & $\mathrm{A}, \mathrm{C}, \mathrm{M}$ & C $100,431,474$ \\
\hline A. pazcuarensis (Kunth) R.M.King. et H.Rob. & $\operatorname{Ar}$ & C & C 153 \\
\hline A. pichinchensis (Kunth) R.M.King et H.Rob. & $\mathrm{H}$ & $\mathrm{R}$ & C 402 \\
\hline Ageratum corymbosum Zuccagni ex Pers. & $\operatorname{Ar}$ & Q & C 391, R 42216 \\
\hline Alloispermum scabrum (Lag.) H.Rob. & $\mathrm{H}$ & Q & C 373, P 2523 \\
\hline Archibaccharis hieracioides (S.F.Blake) S.F.Blake * & $\mathrm{H}$ & $\mathrm{A}, \mathrm{C}$ & C $129,432, \mathrm{R} 48348$ \\
\hline A. hirtella (DC.) Heering & $\operatorname{Ar}$ & A & C 469,471 \\
\hline A. serratifolia (Kunth) S.F.Blake & $\mathrm{H}$ & Q & C 456 \\
\hline Artemisia ludoviciana Nutt. & $\mathrm{H}$ & $\mathrm{A}, \mathrm{Q}$ & C 392, F 127 \\
\hline Aster moranensis Kunth var. moranensis & $\mathrm{H}$ & Q & C 460 \\
\hline Baccharis conferta Kunth & Ar & A, C & C 108,158 \\
\hline B. heterophylla Kunth & $\operatorname{Ar}$ & $\mathrm{MB}$ & C 492 \\
\hline B. multiflora Kunth var. multiflora * & $\mathrm{H}$ & A & C 327, J 6948 \\
\hline Barkleyanthus salicifolius (Kunth) H.Rob. et Brettell & Ar & A & C 109, F 45, \\
\hline Bidens anthemoides (DC.) Sherff * & $\mathrm{H}$ & $\mathrm{A}, \mathrm{C}$ & C $110,412, \mathrm{R} 46030$ \\
\hline B. odorata Cav. var. rosea (Sch.Bip.) Melchert & $\mathrm{H}$ & Q & C 342 \\
\hline B. ostruthioides (DC.) Sch.Bip. & $\mathrm{H}$ & $\mathrm{A}, \mathrm{C}, \mathrm{Q}$ & C 128, S 1710 \\
\hline B. triplinervia Kunth var. triplinervia & $\mathrm{H}$ & A, C & C $56,220,279,435$ \\
\hline Brickellia nutanticeps S.F.Blake & $\mathrm{H}$ & A & C 466 \\
\hline B. pedunculosa (DC.) Harc. et Beaman * & $\mathrm{H}$ & $\mathrm{A}, \mathrm{Q}$ & C 129,457 \\
\hline B. secundiflora (Lag.) A.Gray & $\mathrm{H}$ & $\mathrm{A}, \mathrm{Q}$ & C 454 \\
\hline B. squarrosa B.L.Rob. et Greenm. & $\mathrm{H}$ & C & F 56 \\
\hline Chromolepis heterophylla Benth. * & $\mathrm{H}$ & MJ & C 293 \\
\hline Cirsium anartiolepis Petr. * & $\mathrm{H}$ & $\mathrm{C}$ & F 91, R 42444 \\
\hline C. ehrenbergii Sch.Bip. ${ }^{*}$ & $\mathrm{H}$ & A & E 1 \\
\hline C. jorullense (Kunth) Spreng. subsp. jorullense * & $\mathrm{H}$ & $\mathrm{C}$ & C 32 \\
\hline C. subcoriaceum (Less.) Sch.Bip. & $\mathrm{H}$ & C & C 141, G 3836, \\
\hline Conyza coronopifolia Kunth & $\mathrm{H}$ & A & C 405, F 163, \\
\hline Cosmos bipinnatus Cav. & $\mathrm{H}$ & $\mathrm{R}$ & C 356 \\
\hline C. parviflorus (Jacq.) Kunth & $\mathrm{H}$ & Q & C 343 \\
\hline C. scabiosoides Kunth * & $\mathrm{H}$ & Q & C 360 \\
\hline
\end{tabular}


Guadalupe Cornejo-Tenorio et $A L$.

\begin{tabular}{|c|c|c|c|}
\hline Taxa & FC & TV & $\mathrm{C}$ \\
\hline Cotula mexicana (DC.) Cabrera & $\mathrm{H}$ & C & R 47394, 47811 \\
\hline Dahlia coccinea Cav. & $\mathrm{H}$ & Q & C 374 \\
\hline D. rudis P.D.Sorensen * & $\mathrm{H}$ & M & C 305 \\
\hline D. scapigera (A.Dietr.) Knowles et Westc.* & $\mathrm{H}$ & A & C 246 \\
\hline Dyssodia papposa (Vent.) Hitchc. & $\mathrm{H}$ & Q & C 397 \\
\hline D. pinnata (Cav.) B.L.Rob. var. pinnata* & $\mathrm{H}$ & Q & C 396 \\
\hline Erigeron galeottii (Hemsl.) Greene * & $\mathrm{H}$ & A & C 240,255 \\
\hline E. karvinskianus DC. & $\mathrm{H}$ & $\mathrm{C}$ & F 180 \\
\hline E. longipes DC. & $\mathrm{H}$ & $\mathrm{C}$ & F 179 \\
\hline Galinsoga quadriradiata Ruiz et Pavón & $\mathrm{H}$ & Q & C 380 \\
\hline Gamochaeta americana (Mill.) Cabrera & $\mathrm{H}$ & $\mathrm{C}, \mathrm{P}$ & M 25, T 13056 \\
\hline Gnaphaliothamnus salicifolius (Bertol.) G.L.Nesom & $\mathrm{H}$ & C & C 448, R 48252 \\
\hline Helenium scorzonerifolium (DC.) A.Gray & $\mathrm{H}$ & $A, M J, P$ & C 16, R 52774 \\
\hline Heliopsis procumbens Hemsl. * & $\mathrm{H}$ & A & C 411 \\
\hline Hieracium crepidispermum Fr. & $\mathrm{H}$ & $\mathrm{P}$ & C 313, R 47378 \\
\hline H. dysonymum S.F.Blake * & $\mathrm{H}$ & $\mathrm{C}$ & C 275 \\
\hline Jaegeria glabra (S.Wats.) Rob. * & $\mathrm{H}$ & $\mathrm{P}$ & C 420 \\
\hline J. hirta (Lag.) Less. & $\mathrm{H}$ & $\mathrm{A}, \mathrm{C}, \mathrm{Q}$ & C 58,387, R 48273 \\
\hline Laennecia schiedeana (Less.) G.L.Nesom & $\mathrm{H}$ & A & C 329,429 \\
\hline Melampodium divaricatum (Rich.) DC. & $\mathrm{H}$ & $\mathrm{C}$ & F 214 \\
\hline M. perfoliatum (Cav.) Kunth & $\mathrm{H}$ & $\mathrm{C}$ & F 293 \\
\hline Mexerion sarmentosum (Klatt) G.L.Nesom * & $\mathrm{H}$ & $A, P$ & C 318, R 52772 \\
\hline Montanoa grandiflora DC. * & $\mathrm{Ar}$ & Q & C 348 \\
\hline Osbertia stolonifera (DC.) Greene & $\mathrm{H}$ & $\mathrm{C}, \mathrm{MB}$ & C 195,271 \\
\hline Packera bellidifolia (Kunth) W.A.Weber et A.Löve * & $\mathrm{H}$ & $A, C, P$ & C $312, \mathrm{H} \mathrm{S} / \mathrm{N}$ \\
\hline P. sanguisorbae (DC.) C.Jeffrey* & $\mathrm{H}$ & A & C 59 \\
\hline Pinaropappus roseus (Less.) Less. var. roseus & $\mathrm{H}$ & $\mathrm{C}$ & F 162, MM 89 \\
\hline Piqueria pilosa Kunth * & $\mathrm{H}$ & A, C & C $20,105,228,430$ \\
\hline P. trinervia Cav. & $\mathrm{H}$ & A & C 347 \\
\hline Psacalium peltatum (Kunth) Cass. ${ }^{*}$ & $\mathrm{H}$ & A & $\mathrm{S} / \mathrm{C}$ \\
\hline Pseudognaphalium oxyphyllum (DC.) Kirp. var. oxyphyllum & $\mathrm{H}$ & $\mathrm{C}$ & C 446 \\
\hline P. viscosum (Kunth) Anderb. & $\mathrm{H}$ & $\mathrm{C}$ & C 442,447 \\
\hline Roldana albonervia (Greenm.) H.Rob. et Brettell * & $\mathrm{Ar}$ & A, C & C 15, 96, R 48364 \\
\hline R. angulifolia (DC.) H.Rob. et Brettell & $\mathrm{Ar}$ & A, C, M & C $117,178,424$ \\
\hline R. barba-johannis (DC.) H.Rob. et Brettell & $\mathrm{Ar}$ & $A, C, M, R$ & C 99, 191, S 1958 \\
\hline R. lineolata (DC.) H.Rob. et Brettell * & $\mathrm{H}$ & A, C, Q & C 15,414, P 2553 \\
\hline Rumfordia floribunda DC. * & $\mathrm{Ar}$ & $\mathrm{R}$ & $\mathrm{MS} / \mathrm{N}$ \\
\hline Sabazia humilis (Kunth) Cass. * & $\mathrm{H}$ & M & C 288 \\
\hline $\begin{array}{l}\text { Schkuhria pinnata (Lam.) Kuntze var. wislizenii (A.Gray) } \\
\text { B.L.Turner }\end{array}$ & $\mathrm{H}$ & Q & C 349 \\
\hline Senecio callosus Sch.Bip. & $\mathrm{H}$ & $A, C, M$ & C $103,181,423$ \\
\hline S. cinerarioides Kunth * & $\mathrm{Ar}$ & A, C & C $107, \mathrm{M} \mathrm{S} / \mathrm{N}$ \\
\hline S. helodes Benth. * & $\mathrm{H}$ & $\mathrm{P}$ & C 418 \\
\hline S. stoechadiformis DC. * & $\mathrm{H}$ & $\mathrm{C}, \mathrm{Q}$ & C 143, 449 \\
\hline S. toluccanus DC. * & $\mathrm{H}$ & A, C & C $118, F 27$ \\
\hline S. vulgaris L. & $\mathrm{H}$ & C & C 444 \\
\hline Sigesbeckia jorullensis Kunth & $\mathrm{H}$ & $A, C$ & C $70,238,327$ \\
\hline Simsia amplexicaulis (Cav.) Pers. & $\mathrm{H}$ & C & $\mathrm{MS} / \mathrm{N}$ \\
\hline Sonchus oleraceus L. & $\mathrm{H}$ & $A, C$ & C $215,443, \mathrm{M} \mathrm{S} / \mathrm{N}$ \\
\hline Stevia clinopodioides Greenm. * & $\mathrm{H}$ & A & C 310 \\
\hline S. jorullensis Kunth & $\mathrm{H}$ & $\mathrm{C}$ & C $378,401,415$ \\
\hline S. lucida Lag. var. lucida & $\mathrm{H}$ & C & F 25, 49, P 2552 \\
\hline S. monardifolia Kunth * & $\mathrm{H}$ & $A, C$ & C 125,436 \\
\hline S. origanoides Kunth * & $\mathrm{H}$ & $\mathrm{C}$ & F 297 \\
\hline S. salicifolia Cav.var salicifolia & $\mathrm{Ar}$ & C, Q & C $280,389,450$ \\
\hline S. serrata Cav. var. serrata & $\mathrm{H}$ & C, Q & C 339, F 296 \\
\hline
\end{tabular}




\begin{tabular}{|c|c|c|c|}
\hline Taxa & FC & TV & $\mathrm{C}$ \\
\hline S. subpubescens Lag. var. intermedia Grashoff* & $\mathrm{Ar}$ & $\mathrm{C}$ & F 13, 288 \\
\hline Tagetes erecta L. & $\mathrm{H}$ & Q & C $335, F 192$ \\
\hline T. foetidissima DC. & $\mathrm{H}$ & A & C 328 \\
\hline T. lucida Cav. & $\mathrm{H}$ & $\mathrm{P}, \mathrm{Q}$ & C 385, M 34 \\
\hline T. lunulata Ortega * & $\mathrm{H}$ & $\mathrm{C}$ & A S/N \\
\hline T. micrantha Cav. & $\mathrm{H}$ & Q & C 337 \\
\hline Taraxacum officinale Weber ex F.H.Wigg. & $\mathrm{H}$ & A & C 34 \\
\hline Telanthophora andrieuxii (DC.) H.Rob. et Brettell * & $\mathrm{Ar}$ & $\mathrm{C}$ & M S/N, R 48365 \\
\hline Verbesina klattii B.L.Rob. et Greenm. * & $\mathrm{Ar}$ & M & F 359, T 6926 \\
\hline V. oncophora B.L.Rob. et Seaton * & $\mathrm{Ar}$ & $A, C, M$ & C $93,190,440$ \\
\hline Viguiera hemsleyana Blake * & $\mathrm{H}$ & Q & C 377 \\
\hline \multicolumn{4}{|l|}{ BETULACEAE } \\
\hline Alnus acuminata Kunth subsp. arguta (Schltdl.) Furlow & A & $\mathrm{C}$ & $\mathrm{F} 54, \mathrm{M} \mathrm{S} / \mathrm{N}$ \\
\hline A. jorullensis Kunth subsp. jorullensis & A & $\mathrm{C}$ & C 159 \\
\hline \multicolumn{4}{|l|}{ BORAGINACEAE } \\
\hline Hackelia mexicana (Schltdl. et Cham.) I.M.Johnst. & $\mathrm{H}$ & A & C 74 \\
\hline Lithospermum distichum Ortega & $\mathrm{H}$ & A & C $40, N 10$ \\
\hline L. strictum Lehm. * & $\mathrm{H}$ & A & C 233 \\
\hline \multicolumn{4}{|l|}{ BRASSICACEAE } \\
\hline Brassica rapa L. & $\mathrm{H}$ & $\mathrm{P}$ & C 241 \\
\hline Capsella bursa-pastoris (L.) Medik. & $\mathrm{H}$ & $\mathrm{P}$ & C 323 \\
\hline Cardamine flaccida Cham. et Schltdl. & $\mathrm{H}$ & A & AG 8, C 28 \\
\hline Descurainia impatiens (Cham. et Schltdl.) O.E.Schulz & $\mathrm{H}$ & A & C 203 \\
\hline Eruca sativa Mill. & $\mathrm{H}$ & $\mathrm{P}$ & C 242 \\
\hline Lepidium sordidum A.Gray & $\mathrm{H}$ & A & E 9 \\
\hline L. virginicum $\mathrm{L}$. & $\mathrm{H}$ & $\mathrm{R}$ & F 106 \\
\hline Pennellia longifolia (Benth.) Rollins & $\mathrm{H}$ & A & C 307 \\
\hline Romanschulzia arabiformis (DC.) Rollins & $\mathrm{H}$ & A & C 229 \\
\hline Rorippa nasturtium-aquaticum (L.) Schinz et Thell. & $\mathrm{H}$ & $\mathrm{C}$ & $\mathrm{F} 33, \mathrm{MS} / \mathrm{N}$ \\
\hline \multicolumn{4}{|l|}{ BUDDLEJACEAE } \\
\hline Buddleia cordata Kunth & A & $\mathrm{A}, \mathrm{C}$ & A 6, F 48 \\
\hline B. parviflora Kunth * & A & A & AG 6, E 14 \\
\hline B. sessiliflora Kunth & $\mathrm{Ar}$ & C & C 161 \\
\hline \multicolumn{4}{|l|}{ CALLITRICHACEAE } \\
\hline Callitriche heterophylla Pursh & $\mathrm{H}$ & $\mathrm{C}$ & $M S / N$ \\
\hline \multicolumn{4}{|l|}{ CAMPANULACEAE } \\
\hline Diastatea micrantha (Kunth) McVaugh & $\mathrm{H}$ & Q & C S/N, P 2550 \\
\hline Lobelia cardinalis L. & $\mathrm{H}$ & A & C 51 \\
\hline L. gruina Cav. var. gruina* & $\mathrm{H}$ & Q & C 484, P 2522 \\
\hline L. laxiflora Kunth var. laxiflora & $\mathrm{H}$ & $C, M, R$ & C 174, F 44 \\
\hline L. nana Kunth & $\mathrm{H}$ & MJ & C 295 \\
\hline L. plebeia Wimm. * & $\mathrm{H}$ & $\mathrm{C}$ & R 48512, 53306 \\
\hline \multicolumn{4}{|l|}{ CAPRIFOLIACEAE } \\
\hline Sambucus nigra L. var. canadensis (L.) Bolli. & A & A & C 4, 78, N 1 \\
\hline Symphoricarpos microphyllus Kunth & $\mathrm{Ar}$ & $A, R$ & C 209, Z 5794 \\
\hline Viburnum acutifolium Benth. & $\mathrm{Ar}$ & M & C 185 \\
\hline V. microphyllum (Oerst.) Hemsl. * & $\mathrm{Ar}$ & $\mathrm{C}$ & R 46243, Z 5463 \\
\hline \multicolumn{4}{|l|}{ CARYOPHYLLACEAE } \\
\hline Arenaria bourgaei Hemsl. & $\mathrm{H}$ & A & C 230 \\
\hline
\end{tabular}




\begin{tabular}{|c|c|c|c|}
\hline Taxa & FC & TV & $\mathrm{C}$ \\
\hline A. lanuginosa (Michx.) Rohrb. & $\mathrm{H}$ & C & C 283,286 \\
\hline Cerastium nutans Raf. & $\mathrm{H}$ & A, C & C 27, F 252 \\
\hline C. vulcanicum Schltdl. & $\mathrm{H}$ & $\mathrm{A}, \mathrm{MB}$ & C 257,273 \\
\hline Cerdia congestiflora Hemsl. * & $\mathrm{H}$ & $A, C, V$ & C 87, F 148 \\
\hline Drymaria cordata (L.) Willd. ex Schult. & $\mathrm{H}$ & C & F 32 \\
\hline D. effusa A.Gray & $\mathrm{H}$ & $\mathrm{C}$ & C 244, Z 4492 \\
\hline D. excisa Standl. * & $\mathrm{H}$ & C & F 88 \\
\hline D. malachioides Briq. * & $\mathrm{H}$ & A, M & C 173, L 241 \\
\hline Stellaria cuspidata Willd. ex Schltdl. & $\mathrm{H}$ & A, C & F 92, E 2, P 2536 \\
\hline \multicolumn{4}{|l|}{ CISTACEAE } \\
\hline Helianthemum glomeratum Lag. ex DC. & $\mathrm{H}$ & $\mathrm{Q}, \mathrm{V}$ & C 352,461 \\
\hline \multicolumn{4}{|l|}{ CLETHRACEAE } \\
\hline Clethra mexicana DC. & A & A, M, Q & C 194, 479 \\
\hline \multicolumn{4}{|l|}{ CLUSIACEAE } \\
\hline Hypericum philonotis Schltdl. et Cham. & $\mathrm{H}$ & A, MJ & C 264,285 \\
\hline H. silenoides Juss. var. silenoides & $\mathrm{H}$ & $\mathrm{Q}, \mathrm{MJ}$ & C 297,336 \\
\hline \multicolumn{4}{|l|}{ CONVOLVULACEAE } \\
\hline Ipomoea emetica Choisy* & $\mathrm{H}$ & Q & C 334 \\
\hline \multicolumn{4}{|l|}{ CORNACEAE } \\
\hline Cornus disciflora DC. & A & A, C & F 340, H 3589 \\
\hline \multicolumn{4}{|l|}{ CRASSULACEAE } \\
\hline Altamiranoa mexicana (Schltdl.) Rose & $\mathrm{H}$ & A, Q & R 45818, S 1930 \\
\hline Echeveria secunda Booth ex Lindl. * & $\mathrm{H}$ & $\mathrm{A}, \mathrm{C}$ & C $52,250,496$ \\
\hline Sedum bourgaei Hemsl. * & $\mathrm{H}$ & C, Q & C $403,413,452$ \\
\hline S. minimum Rose * & $\mathrm{H}$ & $\mathrm{C}$ & C 254 \\
\hline \multicolumn{4}{|l|}{ CUCURBITACEAE } \\
\hline Cyclanthera ribiflora (Schltdl.) Cogn. & $\mathrm{Ar}$ & $\mathrm{M}, \mathrm{C}$ & C 186, F 84 \\
\hline \multicolumn{4}{|l|}{ CHENOPODIACEAE } \\
\hline Chenopodium ambrosoides L. & $\mathrm{H}$ & V & F 135 \\
\hline \multicolumn{4}{|l|}{ ERICACEAE } \\
\hline Arbutus xalapensis Kunth & A & $A, C$ & C 134,299, L 248 \\
\hline Arctostaphylos pungens Kunth & $\mathrm{Ar}$ & Q & C 355,458 \\
\hline Comarostaphylis discolor (Hook.) Diggs & $\operatorname{Ar}$ & Q & C 478 \\
\hline C. rupestris (B.L.Rob. et Seaton) Small * & A & A & C $122,428, F 70$ \\
\hline C. longifolia (Benth.) Klotzsch * & A & $A, M$ & A 7, C 5, 176 \\
\hline \multicolumn{4}{|l|}{ EUPHORBIACEAE } \\
\hline Euphorbia dentata Michx. & $\mathrm{H}$ & $\mathrm{R}$ & F 287 \\
\hline E. furcillata Kunth var. furcillata & $\mathrm{H}$ & A & C 13,131 \\
\hline \multicolumn{4}{|l|}{ FABACEAE } \\
\hline Astragalus lyonnetii Barneby * & $\mathrm{H}$ & C & F 186 \\
\hline A. micranthus Desv. var. micranthus* & $\mathrm{H}$ & A & C 33 \\
\hline A. oxyrrhynchus Hemsl. * & $\mathrm{H}$ & MJ & C 263 \\
\hline Cologania biloba (Lindl.) G.Nicholson & $\mathrm{H}$ & Q & C 383 \\
\hline Dalea bicolor Humb. et Bonpl. ex Willd. var. bicolor* & $\mathrm{Ar}$ & Q & C 459 \\
\hline D. thouinii Schrank* & $\mathrm{H}$ & $\mathrm{R}$ & F 290 \\
\hline
\end{tabular}




\begin{tabular}{|c|c|c|c|}
\hline Taxa & FC & TV & $\mathrm{C}$ \\
\hline Desmodium aparines (Link) DC. & $\mathrm{H}$ & $\mathrm{R}$ & F 289 \\
\hline Lupinus bilineatus Benth. & $\mathrm{H}$ & A & C 268 \\
\hline L. elegans Kunth & $\mathrm{H}$ & $\mathrm{A}, \mathrm{C}$ & C 1,61, F 97 \\
\hline L. montanus Kunth & $\mathrm{H}$ & $A, M$ & C 189,425 \\
\hline L. splendens Rose* & $\mathrm{H}$ & A & C 256 \\
\hline Phaseolus coccineus $\mathrm{L}$. & $\mathrm{H}$ & $\mathrm{R}$ & F 261 \\
\hline Phaseolus parviflorus Maréchal, Masch. et Stain & $\mathrm{H}$ & Q & C 384 \\
\hline Trifolium amabile Kunth & $\mathrm{H}$ & $A, P, V$ & F 321, Z 5788 \\
\hline T. mexicanum Hemsl. & $\mathrm{H}$ & A & C 37 \\
\hline T. wormskioldii Lehm. var. ortegae (Greene) Barneby & $\mathrm{H}$ & A & C 75 \\
\hline Vicia americana Muhl. ex Willd. & $\mathrm{H}$ & A & C 89 \\
\hline \multicolumn{4}{|l|}{ FAGACEAE } \\
\hline Quercus castanea Née* & A & Q & C 358 \\
\hline Q. crassifolia Humb. et Bonpl. & A & $\mathrm{Q}$ & F 203 \\
\hline Q. laurina Humb. et Bonpl. & A & A, C, M, Q & C $192,439,488$ \\
\hline Q. obtusata Humb. et Bonpl. * & A & A, Q & C $341,381,438$ \\
\hline Q. rugosa Neé & A & $C, Q$ & P 2531 \\
\hline \multicolumn{4}{|l|}{ GARRYACEAE } \\
\hline Garrya laurifolia Hartw. ex Benth. & A & A & C 6,138 \\
\hline \multicolumn{4}{|l|}{ GENTIANACEAE } \\
\hline Gentiana bicuspidata (G.Don) Briq. * & $\mathrm{H}$ & A & J 6966, M S/N \\
\hline G. spathacea Kunth * & $\mathrm{H}$ & $A, Q$ & C 482, H 3601 \\
\hline G. amarella (L.) Borner subsp. hartwegii (Benth.) J.M.Gillett* & $\mathrm{H}$ & $\mathrm{A}, \mathrm{MJ}$ & C $416, \mathrm{M} \mathrm{S} / \mathrm{N}$ \\
\hline G. amarella (L.) Borner subsp. mexicana (Griseb.) J.M.Gillett * & $\mathrm{H}$ & $A, M J, P$ & C 316, R 47364 \\
\hline Halenia brevicornis (Kunth) G.Don & $\mathrm{H}$ & MJ, P & C 315, F 351 \\
\hline H. plantaginea (Kunth) Griseb. * & $\mathrm{H}$ & A & C 200, P 2565 \\
\hline H. pringlei B.L.Rob. et Seaton * & $\mathrm{H}$ & A & L 249 \\
\hline \multicolumn{4}{|l|}{ GERANIACEAE } \\
\hline Erodium cicutarium (L.) L’Hér. ex Aiton & $\mathrm{H}$ & A & C 115 \\
\hline Geranium cruceroense Knuth * & $\mathrm{H}$ & $\mathrm{C}$ & R 52780 \\
\hline G. deltoideum Rydb. * & $\mathrm{H}$ & A & C 493 \\
\hline G. latum Small * & $\mathrm{H}$ & A & R 52746 \\
\hline G. Iilacinum Knuth * & $\mathrm{H}$ & $\mathrm{A}, \mathrm{C}$ & C 92,124, R 48255 \\
\hline G. potentillaefolium DC. * & $\mathrm{H}$ & A & C $23, \times 4340$ \\
\hline G. seemannii Peyr. & $\mathrm{H}$ & $\mathrm{A}, \mathrm{C}, \mathrm{MB}$ & C $35,272, F 39$ \\
\hline \multicolumn{4}{|l|}{ GROSSULARIACEAE } \\
\hline Ribes affine Kunth * & $\operatorname{Ar}$ & $A, C, M J$ & C 116, 292 \\
\hline \multicolumn{4}{|l|}{ HYDROPHYLLACEAE } \\
\hline Nama prostratum Brand * & $\mathrm{H}$ & $A, M, C$ & C 172, F 80 \\
\hline Phacelia platycarpa (Cav.) Spreng. & $\mathrm{H}$ & $\mathrm{A}, \mathrm{C}$ & C 60, F 183, L 242 \\
\hline \multicolumn{4}{|l|}{ LAMIACEAE } \\
\hline Lepechinia caulescens (Ortega) Epling & $\mathrm{H}$ & $\mathrm{A}, \mathrm{V}$ & C 42, F 318 \\
\hline Prunella vulgaris $\mathrm{L}$. & $\mathrm{H}$ & $A, C$ & C 66, F 237 \\
\hline Salvia concolor Lamb. ex Benth. * & $\mathrm{H}$ & C & F 244 \\
\hline S. elegans Vahl* & $\mathrm{H}$ & $A, C, R$ & C $90,151,427$ \\
\hline S. fulgens Cav. * & $\mathrm{H}$ & $\mathrm{A}, \mathrm{C}$ & C $19,104,165,274$ \\
\hline S. gracilis Benth. & $\mathrm{H}$ & $A, C$ & C 183,259 \\
\hline S. helianthemifolia Benth. * & $\mathrm{H}$ & $A, C$ & F 3 \\
\hline S. lavanduloides Benth. & $\mathrm{H}$ & $A, C$ & C 150,376 \\
\hline
\end{tabular}




\begin{tabular}{|c|c|c|c|}
\hline Taxa & FC & TV & $\mathrm{C}$ \\
\hline S. mexicana L. var. minor Benth. * & $\mathrm{H}$ & $A, M, Q, V$ & C $95,168,183$ \\
\hline S. mexicana L. var. mexicana* & $\mathrm{H}$ & $\mathrm{Q}, \mathrm{P}$ & C 330,331 \\
\hline S. microphylla Kunth & $\mathrm{H}$ & $\mathrm{C}$ & Z 3961 \\
\hline S. patens Cav. * & $\mathrm{H}$ & Q & C 362 \\
\hline S. polystachya Ortega & $\mathrm{H}$ & $\mathrm{Q}, \mathrm{V}$ & C 91,332, Z 4966 \\
\hline S. prunelloides Kunth * & $\mathrm{H}$ & A & C 22,485 \\
\hline S. stricta Sessé et Moc. * & $\mathrm{H}$ & A & C 126,434 \\
\hline Satureja macrostema (Benth.) Briq. & $\mathrm{Ar}$ & $A, M$ & C $101,187,422$ \\
\hline Scutellaria caerulea Sessé et Moc. & $\mathrm{H}$ & $\mathrm{Q}$ & C 375, P 2525 \\
\hline Stachys coccinea Jacq. & $\mathrm{H}$ & $A, C$ & C 97,169 \\
\hline S. parvifolia M.Martens et Galeotti * & $\mathrm{H}$ & A & C 72 \\
\hline S. radicans Epling & $\mathrm{H}$ & $\mathrm{P}$ & C 235 \\
\hline S. sanchezii Rzed. et A.García * & $\mathrm{H}$ & A & C 71, E 3 \\
\hline \multicolumn{4}{|l|}{ LENTIBURALIACEAE } \\
\hline Pinguicula moranensis Kunth & $\mathrm{H}$ & C, Q & C 370, Z 3942 \\
\hline \multicolumn{4}{|l|}{ LINACEAE } \\
\hline Linum orizabae Planch. & $\mathrm{H}$ & $\mathrm{C}$ & R 48251 \\
\hline \multicolumn{4}{|l|}{ LYTHRACEAE } \\
\hline Cuphea aequipetala Cav. & $\mathrm{H}$ & $\mathrm{A}, \mathrm{C}$ & C $38,372, F 36$ \\
\hline C. jorullensis Kunth * & $\mathrm{H}$ & $\mathrm{C}$ & C 145, F 222 \\
\hline \multicolumn{4}{|l|}{ MALVACEAE } \\
\hline Kearnemalvastrum subtriflorum (Lag.) D.M.Bates & $\mathrm{H}$ & $A, R$ & F 285, M S/N \\
\hline Malva parviflora L. & $\mathrm{H}$ & $\mathrm{R}$ & F 305 \\
\hline \multicolumn{4}{|l|}{ MONOTROPACEAE } \\
\hline Monotropa hypopitys L. & $\mathrm{H}$ & A & C 202 \\
\hline M. uniflora L. & $\mathrm{H}$ & A & C 17,201 \\
\hline \multicolumn{4}{|l|}{ ONAGRACEAE } \\
\hline Epilobium ciliatum Raf. & $\mathrm{H}$ & A & C 62 \\
\hline Fuchsia microphylla Kunth & $\mathrm{Ar}$ & A & C $10,139,437$ \\
\hline F. thymifolia Kunth & $\mathrm{Ar}$ & $A, C$ & C 207,281 \\
\hline Lopezia racemosa Cav. & $\mathrm{H}$ & $A, C, Q, V$ & C 166, 395 \\
\hline Oenothera pubescens Willd. ex Spreng. & $\mathrm{H}$ & A & C 219 \\
\hline O. purpusii Munz * & $\mathrm{H}$ & $\mathrm{A}, \mathrm{C}$ & EP 262, F 157 \\
\hline O. rosea L'Hér. ex Aiton & $\mathrm{H}$ & A & C 2 \\
\hline \multicolumn{4}{|l|}{ OROBANCHACEAE } \\
\hline Conopholis alpina Liebm. & $\mathrm{P}$ & Q & Z 7271 \\
\hline \multicolumn{4}{|l|}{ OXALIDACEAE } \\
\hline Oxalis alpina (Rose) Kunth & $\mathrm{H}$ & $\mathrm{A}, \mathrm{C}$ & C 30,494 \\
\hline O. corniculata $\mathrm{L}$. & $\mathrm{H}$ & A & C 36 \\
\hline \multicolumn{4}{|l|}{ PAPAVERACEAE } \\
\hline Argemone platyceras Link et Otto & $\mathrm{H}$ & A & C 55 \\
\hline Bocconia frutescens $\mathrm{L}$. & $\operatorname{Ar}$ & $C, M$ & C 188, Z 7406 \\
\hline \multicolumn{4}{|l|}{ PHYTOLACCACEAE } \\
\hline Phytolacca icosandra L. & $\mathrm{H}$ & $A, V$ & C $14,149,441$ \\
\hline \multicolumn{4}{|l|}{ PIPERACEAE } \\
\hline Peperomia campylotropa A.W.Hill. & $\mathrm{H}$ & $\mathrm{A}, \mathrm{C}, \mathrm{MJ}$ & C 495, Z 3929 \\
\hline
\end{tabular}




\begin{tabular}{|c|c|c|c|}
\hline Taxa & FC & TV & $\mathrm{C}$ \\
\hline P. galioides Kunth & $\mathrm{H}$ & $\mathrm{V}$ & F 331, P 2529 \\
\hline P. hispidula (Sw.) A.Dietr. & $\mathrm{H}$ & A & H 3586, R 48506 \\
\hline P. quadrifolia (L.) Kunth & $\mathrm{E}$ & $\mathrm{C}$ & R 46242 \\
\hline \multicolumn{4}{|l|}{ PLANTAGINACEAE } \\
\hline Plantago australis Lam. & $\mathrm{H}$ & $\mathrm{A}, \mathrm{V}$ & C 69, E 5 \\
\hline P. nivea Kunth & $\mathrm{H}$ & A & C 39 \\
\hline \multicolumn{4}{|l|}{ POLEMONIACEAE } \\
\hline Loeselia mexicana (Lam.) Brand & $\mathrm{H}$ & Q & C 398,481 \\
\hline Polemonium mexicanum Cerv. ex Lag. ${ }^{*}$ & $\mathrm{H}$ & A & C 222, E 7 \\
\hline \multicolumn{4}{|l|}{ POLYGALACEAE } \\
\hline Monnina ciliolata DC. * & $\operatorname{Ar}$ & C, Q & C 144,477 \\
\hline \multicolumn{4}{|l|}{ POLYGONACEAE } \\
\hline Polygonum aviculare $\mathrm{L}$. & $\mathrm{H}$ & $\mathrm{C}, \mathrm{V}$ & C 445, F 139 \\
\hline P. lapathifolium L. & $\mathrm{H}$ & V & F 308 \\
\hline Rumex acetocella L. & $\mathrm{H}$ & $\mathrm{R}$ & C 502 \\
\hline R. crispus L. & $\mathrm{H}$ & A & C 322 \\
\hline \multicolumn{4}{|l|}{ PORTULACACEAE } \\
\hline $\begin{array}{l}\text { Claytonia perfoliata Donn var. mexicana (Rydb.) Mill. } \\
\text { et Chambers }\end{array}$ & $\mathrm{H}$ & A & C 29,404 \\
\hline \multicolumn{4}{|l|}{ PRIMULACEAE } \\
\hline Anagallis arvensis $\mathrm{L}$. & $\mathrm{H}$ & $A, C$ & C 224, F 95 \\
\hline \multicolumn{4}{|l|}{ PYROLACEAE } \\
\hline Chimaphila umbellata (L.) Barton & $\mathrm{H}$ & A & C 225,284 \\
\hline \multicolumn{4}{|l|}{ RANUNCULACEAE } \\
\hline Clematis dioica $\mathrm{L}$. & $\mathrm{Ar}$ & Q & C $394, F 200,358$ \\
\hline Ranunculus dichotomus Moc. et Sessé ex DC. & $\mathrm{H}$ & $\mathrm{A}, \mathrm{MB}$ & C 226, L 247 \\
\hline R. peruvianus Pers. & $\mathrm{H}$ & MJ & C 296 \\
\hline \multicolumn{4}{|l|}{ RESEDACEAE } \\
\hline Reseda luteola $\mathrm{L}$. & $\mathrm{H}$ & V & F 138 \\
\hline \multicolumn{4}{|l|}{ RHAMNACEAE } \\
\hline Ceanothus coeruleus Lag. & $\operatorname{Ar}$ & $\mathrm{A}, \mathrm{C}, \mathrm{Q}$ & C $132,278,453$ \\
\hline Rhamnus mucronata Schltdl. & A & C & F 198 \\
\hline \multicolumn{4}{|l|}{ ROSACEAE } \\
\hline Acaena elongata L. & $\operatorname{Ar}$ & $A, C$ & C $8,68, F 243$ \\
\hline Alchemilla aphanoides L.f. var. subalpestris (Rose) Perry & $\mathrm{H}$ & $\mathrm{P}$ & C 81 \\
\hline A. pringlei (Rydb.) Fedde & $\mathrm{H}$ & V & F 147 \\
\hline A. procumbens Rose & $\mathrm{H}$ & $\mathrm{A}, \mathrm{C}$ & C 467, F 230 \\
\hline Crataegus mexicana Moc. et Sessé ex DC. & A & C & C 170, F 99 \\
\hline Fragaria mexicana Schltdl. * & $\mathrm{H}$ & $\mathrm{A}, \mathrm{V}$ & F 159, MC 3 \\
\hline Holodiscus pachydiscus (Rydb.) Standl. * & A & $M$ & C 182 \\
\hline Potentilla candicans Humb. et Bonpl. * & $\mathrm{H}$ & A & L 244 \\
\hline P. rubra Willd. ex Schltdl. * & $\mathrm{H}$ & A & C $24, M C 4$ \\
\hline Prunus brachybotrya Zucc. & A & C & F 90 \\
\hline P. serotina Ehrh. & A & $\mathrm{C}, \mathrm{MJ}$ & C 291, F 86 \\
\hline Rubus liebmannii Focke* & $\operatorname{Ar}$ & C & C 155, F 23 \\
\hline R. pumilus Focke * & $\mathrm{H}$ & A & M S/N, R 52747 \\
\hline
\end{tabular}




\begin{tabular}{|c|c|c|c|}
\hline Taxa & FC & TV & C \\
\hline \multicolumn{4}{|l|}{ RUBIACEAE } \\
\hline Bouvardia longiflora (Cav.) Kunth & $\operatorname{Ar}$ & Q & C 338 \\
\hline B. ternifolia (Cav.) Schltdl. & $\mathrm{H}$ & $C, M J, Q$ & C 234,333 \\
\hline Crusea diversifolia (Kunth) W.R.Anderson & $\mathrm{H}$ & Q & C 359 \\
\hline C. longiflora (Willd. ex Roem. et Schult.) W.R.Anderson & $\mathrm{H}$ & $\mathrm{Q}$ & C 386 \\
\hline Didymaea alsinoides (Cham. et Schltdl.) Standl. * & $\mathrm{H}$ & A & C 236, R 48392 \\
\hline D. floribunda Rzed. & $\mathrm{H}$ & C & F 61 \\
\hline Galium aschenbornii Nees et Schauer & $\mathrm{H}$ & $\mathrm{A}, \mathrm{Q}$ & C 208,480 \\
\hline G. mexicanum Kunth & $\mathrm{H}$ & Q & C 393 \\
\hline Hedyotis pygmaea Roem. et Schult. & $\mathrm{H}$ & $\mathrm{P}$ & C 86 \\
\hline \multicolumn{4}{|l|}{ SALICACEAE } \\
\hline Salix paradoxa Kunth * & A & A, C, M & C 184, F 250 \\
\hline \multicolumn{4}{|l|}{ SAXIFRAGACEAE } \\
\hline Heuchera orizabensis Hemsl. * & $\mathrm{H}$ & M & C 171 \\
\hline \multicolumn{4}{|l|}{ SCROPHULARIACEAE } \\
\hline Calceolaria mexicana Benth. & $\mathrm{H}$ & $\mathrm{A}, \mathrm{C}$ & C 11, F 345 \\
\hline Castilleja arvensis Schltdl. et Cham. & $\mathrm{H}$ & A, C & C 102,160 \\
\hline C. lithospermoides Kunth * & $\mathrm{H}$ & A & C 199,497 \\
\hline C. moranensis Kunth * & $\mathrm{H}$ & A & L 245 \\
\hline C. tenuiflora Benth. & $\mathrm{H}$ & $\mathrm{C}$ & C 247 \\
\hline Lamourouxia multifida Kunth & $\mathrm{H}$ & Q & C 364, P 2567 \\
\hline Mecardonia procumbens (Mill.) Small & $\mathrm{H}$ & $\mathrm{Q}$ & C 399 \\
\hline Mimulus glabratus Kunth & $\mathrm{H}$ & $A, C, P$ & C 12, 85 \\
\hline Pedicularis mexicana Zucc. ex Benth. * & $\mathrm{H}$ & $\mathrm{P}, \mathrm{V}$ & C 83, 417 \\
\hline Penstemon campanulatus (Cav.) Willd. & $\mathrm{H}$ & A & C 54, F 337 \\
\hline P. gentianoides (Kunth) Poir. & $\mathrm{H}$ & $M J, P$ & C 317 \\
\hline P. roseus (Cerv. ex Sweet) G.Don * & $\mathrm{H}$ & $\mathrm{C}, \mathrm{Q}$ & C $301,365,470$ \\
\hline Sibthorpia rotundifolia (Ruiz et Pav.) Edwin & $\mathrm{H}$ & A & C 46,426 \\
\hline Veronica americana (Raf.) Schwein. & $\mathrm{H}$ & $\mathrm{A}, \mathrm{C}$ & C 77, F 235 \\
\hline$V$. arvensis $\mathrm{L}$. & $\mathrm{H}$ & A & C 111 \\
\hline V. peregrina L. subsp. xalapensis (Kunth) Pennell & $\mathrm{H}$ & $M B$ & C 204 \\
\hline V. serpyllifolia L. & $\mathrm{H}$ & $\mathrm{MB}$ & C 223 \\
\hline \multicolumn{4}{|l|}{ SOLANACEAE } \\
\hline Cestrum anagyris Dunal & $\mathrm{Ar}$ & A & C 465 \\
\hline C. nitidum M.Martens et Galeotti * & $\operatorname{Ar}$ & $\mathrm{R}$ & F 312, P 2518, 2538 \\
\hline C. roseum Kunth & $\mathrm{Ar}$ & C & C 164 \\
\hline C. thyrsoideum Kunth * & $\operatorname{Ar}$ & $\mathrm{A}, \mathrm{C}, \mathrm{Q}$ & C 9, 45, 325, 388 \\
\hline Jaltomata procumbens (Cav.) J.L.Gentry & $\mathrm{H}$ & $\mathrm{A}, \mathrm{V}$ & E 11, F 263 \\
\hline Lycianthes rzedowski E.Dean * & $\mathrm{Ar}$ & C & F 191 \\
\hline Physalis coztomatl Moc. et Sessé ex Dunal * & Ar & A & C 121, 239 \\
\hline P. volubilis Waterf. * & $\mathrm{H}$ & A, C & E 8, 210 \\
\hline Solanum appendiculatum Humb. et Bonpl. ex Dunal & $\mathrm{H}$ & A & C 146 \\
\hline S. cardiophyllum Lindl. * & $\mathrm{H}$ & V & F 336 \\
\hline S. cervantesii Lag. & $\operatorname{Ar}$ & $\mathrm{A}, \mathrm{C}$ & C $18,167,463$ \\
\hline S. demissum Lindl. & $\mathrm{H}$ & A & A 1, C 57 \\
\hline S. nigrescens M.Martens et Galeotti & $\mathrm{H}$ & $A, M, Q$ & C $3,289,357$ \\
\hline S. nudum Dunal & $\mathrm{H}$ & $\mathrm{C}$ & F 211 \\
\hline \multicolumn{4}{|l|}{ STYRACACEAE } \\
\hline Styrax argenteus C. Presl var. ramirezii (Greenm.) Gonsoulin & A & $\mathrm{C}$ & C 157 \\
\hline \multicolumn{4}{|l|}{ SYMPLOCACEAE } \\
\hline Symplocos citrea Lex. * & A & $\mathrm{C}$ & F 205 \\
\hline
\end{tabular}




\begin{tabular}{|c|c|c|c|}
\hline Taxa & FC & TV & $\mathrm{C}$ \\
\hline \multicolumn{4}{|l|}{ THEACEAE } \\
\hline Cleyera integrifolia (Benth.) Choisy* & A & $\mathrm{C}$ & C 489, F 332 \\
\hline Ternstroemia lineata DC. subsp. lineata * & A & $\mathrm{C}$ & C 148, F 76 \\
\hline \multicolumn{4}{|l|}{ URTICACEAE } \\
\hline Urtica mexicana Liebm. & $\mathrm{H}$ & A & C 79, 98 \\
\hline \multicolumn{4}{|l|}{ VALERIANACEAE } \\
\hline Valeriana barbareifolia M.Martens et Galeotti & $\mathrm{H}$ & $\mathrm{R}$ & F 292 \\
\hline V. clematitis Kunth & $\mathrm{H}$ & A, M & C 177, F 218 \\
\hline V. pulchella M.Martens et Galeotti & $\mathrm{H}$ & A, C & C 26,249 \\
\hline V. vaginata Kunth * & $\mathrm{H}$ & A & MC 2 \\
\hline \multicolumn{4}{|l|}{ VERBENACEAE } \\
\hline Lippia umbellata Cav. & $\mathrm{Ar}$ & $\mathrm{C}$ & C 142, F 58 \\
\hline Verbena bipinnatifida Nutt. & $\mathrm{H}$ & $\mathrm{Q}$ & C 400,455 \\
\hline V. gracilis Desf. & $\mathrm{H}$ & $A, P, V$ & C $80, F 164$ \\
\hline V. litoralis Kunth & $\mathrm{H}$ & V & F 129 \\
\hline V. menthaefolia Benth. & $\mathrm{H}$ & $\mathrm{C}$ & $\mathrm{MS} / \mathrm{N}$ \\
\hline V. recta Kunth * & $\mathrm{H}$ & $A, Q, V$ & C 73,361 \\
\hline \multicolumn{4}{|l|}{ VIOLACEAE } \\
\hline $\begin{array}{l}\text { Hybanthus attenuatus (Humb. et Bonpl. ex Roem. } \\
\text { et Schult.) Schulze-Menz }\end{array}$ & $\mathrm{H}$ & $\mathrm{R}$ & F 341 \\
\hline Viola humilis Kunth & $\mathrm{H}$ & $A, V$ & F 193, R 47368 \\
\hline \multicolumn{4}{|l|}{ VISCACEAE } \\
\hline Arceuthobium abietis-religiosae Heil * & $\mathrm{P}$ & $\mathrm{A}, \mathrm{C}$ & R 38058b, 46011, \\
\hline A. globosum Hawksw. et Wiens. & $\mathrm{P}$ & $\mathrm{A}, \mathrm{C}$ & R 48461, 48462 \\
\hline Cladocolea diversifolia (Benth.) Kuijt & $\mathrm{P}$ & Q & C 340 \\
\hline C. grahamii (Benth.) Tiegh. * & $\mathrm{P}$ & $\mathrm{C}$ & R 42440 \\
\hline Phoradendron schumanni Trel. * & $\mathrm{P}$ & Q & C 371 \\
\hline P. velutinum (DC.) Nutt. & $P$ & $\mathrm{C}$ & F 2 \\
\hline \multicolumn{4}{|l|}{ CLASE LILIOPSIDA } \\
\hline \multicolumn{4}{|l|}{ AGAVACEAE } \\
\hline Furcraea bedinghausii K.Koch * & $\operatorname{Ar}$ & A & $\mathrm{S} / \mathrm{C}$ \\
\hline \multicolumn{4}{|l|}{ ANTHERICACEAE } \\
\hline Echeandia flavescens (Schult. et Schult.f.) Cruden & $\mathrm{H}$ & $P$ & C 298 \\
\hline \multicolumn{4}{|l|}{ BROMELIACEAE } \\
\hline Tillandsia andrieuxii (Mez) L.B.Sm. * & E & Q & C 483 \\
\hline T. erubescens Schltdl. * & E & Q & C 367 \\
\hline T. macdougallii L.B.Sm. * & E & $\mathrm{Q}$ & C 304 \\
\hline T. prodigiosa (Lem.) Baker* & $\mathrm{E}$ & $\mathrm{C}$ & C 486 \\
\hline \multicolumn{4}{|l|}{ CYPERACEAE } \\
\hline Carex peucophila Holm & $\mathrm{H}$ & A & C 231 \\
\hline Cyperus ses/erioides Kunth & $\mathrm{H}$ & $\mathrm{C}$ & C 253 \\
\hline Eleocharis acicularis (L.) Roem. et Schult. & $\mathrm{H}$ & MB & C 210 \\
\hline E. bonariensis Nees & $\mathrm{H}$ & $\mathrm{A}, \mathrm{MB}$ & C $216,267,269$ \\
\hline \multicolumn{4}{|l|}{ COMMELINACEAE } \\
\hline Commelina orchioides Booth ex Lindl. & $\mathrm{H}$ & $\mathrm{Q}, \mathrm{V}$ & C 88, F 320 \\
\hline Gibasis pulchella (Kunth) Raf. & $\mathrm{H}$ & $A, C, Q$ & C $25,276,379$ \\
\hline
\end{tabular}




\begin{tabular}{|c|c|c|c|}
\hline Taxa & $\mathrm{FC}$ & TV & C \\
\hline Tinantia erecta (Jacq.) Schltdl. & $\mathrm{H}$ & $\mathrm{R}$ & F 325 \\
\hline Tradescantia comelinoides Schult. et Schult.f. & $\mathrm{H}$ & $\mathrm{C}$ & F 238 \\
\hline Weldenia candida Schult.f. & $\mathrm{H}$ & A & MC 1 \\
\hline \multicolumn{4}{|l|}{ ERIOCAULACEAE } \\
\hline Eriocaulon benthamii Kunth & $\mathrm{H}$ & MB & C 218 \\
\hline \multicolumn{4}{|l|}{ HYPOXIDACEAE } \\
\hline Hypoxis mexicana Schult. et Schult.f. & $\mathrm{H}$ & MB & C 205 \\
\hline \multicolumn{4}{|l|}{ IRIDACEAE } \\
\hline $\begin{array}{l}\text { Sisyrinchium angustissimum (B.L.Rob. et Greenm.) } \\
\text { Greenm. et C.H.Thomps. * }\end{array}$ & $\mathrm{H}$ & $\mathrm{R}$ & R 44217 \\
\hline Sisyrinchium cernuum (E.P.Bicknell) Kearney & $\mathrm{H}$ & A & C 112 \\
\hline S. convolutum Nocca & $\mathrm{H}$ & $\mathrm{A}, \mathrm{MB}$ & C 63,266 \\
\hline S. conzattii Calderón et Rzed. * & $\mathrm{H}$ & $\mathrm{P}$ & C 303 \\
\hline S. scabrum Schltr. et Cham. & $\mathrm{H}$ & A & C 53 \\
\hline S. schaffneri S.Watson * & $\mathrm{H}$ & MB & C 265 \\
\hline \multicolumn{4}{|l|}{ JUNCACEAE } \\
\hline $\begin{array}{l}\text { Juncus arcticus Willd. var. mexicanus (Willd. ex Schult. } \\
\text { et Schult.f.) Balslev }\end{array}$ & $\mathrm{H}$ & A & C 21,217 \\
\hline J. ebracteatus E.Mey. & $\mathrm{H}$ & MJ & C 419 \\
\hline Luzula caricina E.Mey. & $\mathrm{H}$ & $\mathrm{C}$ & C 270 \\
\hline \multicolumn{4}{|l|}{ MELANTHIACEAE } \\
\hline Stenanthium frigidum (Schltdl. et Cham.) Kunth * & $\mathrm{H}$ & A & $\mathrm{C} 409, \mathrm{M} \mathrm{S} / \mathrm{N}$ \\
\hline \multicolumn{4}{|l|}{ ORCHIDACEAE } \\
\hline Corallorrhiza involuta Greenm. * & $\mathrm{H}$ & C & Z 7404 \\
\hline C. maculata Raf. & $\mathrm{H}$ & $\mathrm{C}$ & Z 7407 \\
\hline Govenia liliacea (La Llave et Lex.) Lindl. & $\mathrm{H}$ & $\mathrm{A}, \mathrm{C}$ & C 206, Z 3954 \\
\hline Habenaria guadalajarana S.Watson & $\mathrm{H}$ & A & C 319 \\
\hline Malaxis soulei L.O.Williams & $\mathrm{H}$ & $\mathrm{A}, \mathrm{C}$ & C 49,248 \\
\hline Prescotia tubulosa (Lindl.) L.O.Williams & $\mathrm{H}$ & C & M 81 \\
\hline Spiranthes hyemalis A.Rich. et Galeotti & $\mathrm{H}$ & A & C 491 \\
\hline \multicolumn{4}{|l|}{ POACEAE } \\
\hline Agrostis bourgaei Fourn. * & $\mathrm{H}$ & A & C 67 \\
\hline Brachypodium mexicanum (Roem. et Schult.) Link. & $\mathrm{H}$ & $\mathrm{C}$ & MM 95 \\
\hline Bromus exaltatus Bernh. & $\mathrm{H}$ & $\mathrm{P}$ & C 282,314 \\
\hline Cinna poaeformis (Kunth) Scribn. et Merr. & $\mathrm{H}$ & MJ & C 324 \\
\hline Festuca subverticillata (Pers.) E.Alexeev & $\mathrm{H}$ & A & C 64 \\
\hline Lycurus phalaroides Kunth & $\mathrm{H}$ & Q & C 351 \\
\hline Piptochaetium fimbriatum (Kunth) Hitchc. & $\mathrm{H}$ & $\mathrm{P}$ & C 260 \\
\hline Poa annua L. & $\mathrm{H}$ & A & C 408 \\
\hline Setaria parviflora (Poir.) Kerguélen & $\mathrm{H}$ & $\mathrm{P}$ & C 262 \\
\hline Trisetum kochianum Hern.Torres & $\mathrm{H}$ & A & C $213,245,410$ \\
\hline T. virletti Fourn. * & $\mathrm{H}$ & A & M 3 \\
\hline Vulpia myurus C.C.Gmel. & $\mathrm{H}$ & A, C & C $211,407,421$ \\
\hline \multicolumn{4}{|l|}{ SMILACACEAE } \\
\hline Smilax moranensis M.Martens et Galeotti * & $\mathrm{H}$ & A & C 7 \\
\hline
\end{tabular}

